\title{
An image-computable model on how endogenous and exogenous attention differentially alter visual perception
}

\author{
Michael Jigo ${ }^{*}$, David J. Heeger ${ }^{1,2} \&$ Marisa Carrasco ${ }^{1,2}$ \\ ${ }^{1}$ Center for Neural Science and ${ }^{2}$ Department of Psychology \\ New York University, New York, NY 10003
}

\section{*Corresponding author information:}

Michael Jigo (michael.jigo@nyu.edu)

\section{Author Contributions}

M.J., D.J.H. and M.C. conceived the project; M.J. implemented the model with input from D.J.H. and M.C.; M.C. provided all the data to be modelled; M.J. wrote the manuscript with guidance and supervision from M.C. All three authors edited the manuscript.

\section{Acknowledgements}

This work was supported by National Institutes of Health RO1-EY019693 to M.C. We thank Michael Landy, Jonathan Winawer, Antoine Barbot, Hsin-Hung Li, as well as Antonio Fernández, Nina Hanning, Marc Himmelburg, Luke Huszar \& Yong-Jun Lin and other members of the Carrasco 


\section{ABSTRACT}

Attention alters perception across the visual field. Typically, endogenous (voluntary) and exogenous (involuntary) attention similarly improve performance in many visual tasks, but they

30 have differential effects in some tasks. Extant models of visual attention assume that the effects of

31 these two types of attention are identical and consequently do not explain differences between

32 them. Here, we develop a model of spatial resolution and attention that distinguishes between

33 endogenous and exogenous attention. We focus on texture-based segmentation as a model

34 system because it has revealed a clear dissociation between both attention types. For a texture for

35 which performance peaks at parafoveal locations, endogenous attention improves performance

36 across eccentricity, whereas exogenous attention improves performance where the resolution is

37 low (peripheral locations) but impairs it where the resolution is high (foveal locations) for the scale

38 of the texture. Our model emulates sensory encoding to segment figures from their background

39 and predict behavioral performance. To explain attentional effects, endogenous and exogenous

40 attention require separate operating regimes across visual detail (spatial frequency). Our model

41 reproduces behavioral performance across several experiments and simultaneously resolves three

42 unexplained phenomena: (1) the parafoveal advantage in segmentation, (2) the uniform

43 improvements across eccentricity by endogenous attention and (3) the peripheral improvements

44 and foveal impairments by exogenous attention. Overall, we unveil a computational dissociation

45 between each attention type and provide a generalizable framework for predicting their effects on

46 perception across the visual field. 


\section{INTRODUCTION}

Endogenous and exogenous spatial attention prioritize subsets of visual information and facilitate their processing without concurrent eye movements (1-3). Selection by endogenous attention is goal-driven and adapts to task demands whereas exogenous attention transiently and automatically orients to salient stimuli (1-3). In most visual tasks both types of attention typically improve visual perception similarly (e.g., acuity (4-6), visual search $(7,8)$, perceived contrast (911)). Consequently, models of visual attention do not distinguish between endogenous and exogenous attention (e.g., (12-19)). However, stark differences also exist. Each attention type differentially modulates neural responses $(20,21)$ and fundamental properties of visual processing, including temporal resolution $(22,23)$, texture sensitivity (24), sensory tuning (25), contrast sensitivity (26) and spatial resolution (27-34).

The effects of endogenous and exogenous attention are dissociable during texture segmentation, a visual task constrained by spatial resolution (reviews(1-3)). Whereas endogenous attention optimizes spatial resolution to improve the detection of an attended texture (32-34), exogenous attention reflexively enhances resolution even when detrimental to perception (27-31, 34). Extant models of attention do not explain these well-established effects.

Two main hypotheses have been proposed to explain how attention alters spatial resolution. sensitivity $(30,33)$. Neurophysiological $(13,35,36)$ and neuroimaging $(37,38)$ studies bolster the idea that attention modifies spatial profiles of neural receptive fields (2). Both hypotheses provide qualitative predictions of attentional effects but do not specify their underlying neural computations.

Differences between endogenous and exogenous attention are well established in segmentation tasks and thus provide an ideal model system to uncover their separate roles in altering perception. Texture-based segmentation is a fundamental process of mid-level vision that isolates regions of local structure to extract figures from their background (39-41). Successful segmentation hinges on the overlap between the visual system's spatial resolution and the levels of detail (i.e., SF) encompassed by the texture $(39,41,42)$. Consequently, the ability to distinguish between adjacent textures varies as resolution declines toward the periphery (43-46). Each attention type differentially alters texture segmentation, demonstrating that their effects shape spatial resolution

80 (reviews(1-3)). 
82 Current models of texture segmentation do not explain performance across eccentricity and the

83 distinct modulations by attention. Conventional models treat segmentation as a feedforward

84 process that encodes the elementary features of an image (e.g., SF and orientation), transforms

85 them to reflect the local structure (e.g., regions of similarly oriented bars), then pools across space

86 to emphasize texture-defined contours $(39,41,47)$. Few of these models account for variations in

87 resolution across eccentricity $(46,48,49)$ or endogenous (but not exogenous) attentional

88 modulations $(18,50)$. All others postulate that segmentation is a 'preattentive' (42) operation

89 whose underlying neural processing is impervious to attention (39, 41, 46-49).

Here, we develop a computational model in which feedforward processing and attentional gain contribute to segmentation performance. We augment a conventional model of texture processing $(39,41,47)$. Our model varies with eccentricity and includes contextual modulation within local regions in the stimulus via normalization (51), a canonical neural computation (52). The defining characteristic of normalization is that an individual neuron is (divisively) suppressed by the summed activity of neighboring neurons responsive to different aspects of a stimulus. We model attention as multiplicative gains (attentional gain factors (15)) that vary with eccentricity and SF. Attention shifts sensitivity toward fine or coarse spatial scales depending on the range of SFs enhanced. Our model is image-computable, which allowed us to reproduce behavior directly from grayscale images used in psychophysical experiments $(6,26,27,29-33)$. The model explains three signatures of texture segmentation hitherto unexplained within a single computational framework

104 (Figure 1). (i) The central performance drop (CPD) (27-34, 43-46) (Figure 1A), i.e., the parafoveal

105 advantage of segmentation over the fovea. (ii) The improvements in the periphery and impairments at foveal locations induced by exogenous attention (27-32, 34) (Figure 1B). (iii) The equivalent improvements across eccentricity by endogenous attention (32-34) (Figure 1C).

Whereas our analyses focused on texture segmentation, our model is general and can be applied to other visual phenomena. We show that the model predicts contrast sensitivity across SF and eccentricity as well as the effects of attention on contrast sensitivity and acuity; i.e. in tasks in

112 which both endogenous and exogenous attention have similar or differential effects on

113 performance. To preview our results, model comparisons revealed that normalization is necessary

114 to elicit the CPD and that separate profiles of gain enhancement across SF (26) generate the

115 effects of exogenous and endogenous attention on texture segmentation. A preferential high-SF

116 enhancement reproduces the impairments by exogenous attention due to a shift in visual 
117 sensitivity toward details too fine to distinguish the target at foveal locations. The transition from

118 impairments to improvements in the periphery results from exogenous attentional gain gradually

119 shifting to lower SFs that are more amenable for target detection. Improvements by endogenous

120 attention result from a uniform enhancement of SFs that encompass the target, optimizing visual

121 sensitivity for the attended stimulus across eccentricity.

A

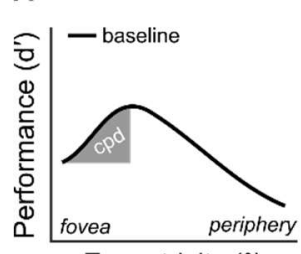

Eccentricity $\left(^{\circ}\right)$
B

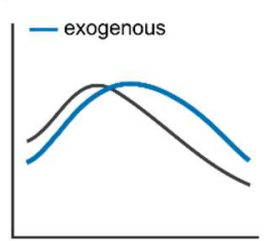

C

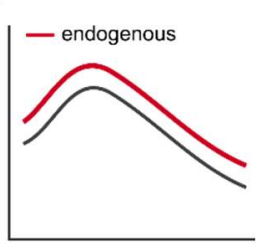

Figure 1. Signatures of texture segmentation.

126 (A) Central performance drop. Shaded region depicts the magnitude of the central performance drop. Identical axis labels are omitted in panels $\mathrm{B}$ and $\mathrm{C}$.

130 (C) Endogenous attention modulation. Endogenous attention improves segmentation performance

(B) Exogenous attention modulation. Exogenous attention improves segmentation performance in the periphery and impairs it near the fovea. across eccentricity. 


\section{RESULTS}

\section{Image-computable model of attention and spatial resolution}

135 We developed an observer model based on established principles of neural computation $(51,52)$,

136 pattern $(53,54)$ and texture vision $(39,41,47)$ and attentional modulation (15). The model

137 incorporates elements of the Reynolds-Heeger normalization model of attention (NMA) (15) and

138 illuminates how attention alters contrast and texture sensitivity across SF and eccentricity. We

139 implement: (i) SF-tuned gain modulation to emulate the decline in contrast sensitivity and peak SF

140 preference with eccentricity. (ii) Spatial summation of normalized inputs to generate texture

141 selectivity. (iii) Separate attentional gain profiles across SF to reproduce effects of exogenous and

142 endogenous attention. The model is composed of four components: stimulus drive, attentional

143 gain, suppressive drive and spatial summation (Figure 2A). Following NMA, attention adjusts the

144 gain on the stimulus drive before normalization. For a full description of the model, see Methods.

146 Stimulus drive. We simulate bottom-up responses of a collection of linear receptive fields (RFs),

147 each jointly tuned to spatial position, SF and orientation. Images are processed through a filter

148 bank (55) covering the visual field at several SFs and orientations using bandwidths compatible

149 with neurophysiological (54) and psychophysical (53) measurements. Filter outputs are combined

150 across quadrature phase (56), yielding contrast energy images corresponding to different SFs and

151 orientations. These outputs simulate the responses of complex cells in primary visual cortex (54,

152 56). The gain on individual RFs varies as a function of SF and eccentricity preference (Figure 2A,

153 green). Following the behavior of individual neurons (54) and pattern vision (53), gain modulation

154 is narrowly tuned to high SFs near the fovea and progressively shifts to low SFs with eccentricity.

155 Consequently, the stimulus drive reflects local spectral energy within each patch in an image,

156 filtered through feature-selective RFs that vary with eccentricity.

Attentional gain. Attention is implemented as a gain control mechanism that scales the gain on the

159 stimulus drive (15). The magnitude of attentional gain is largest at the cued location (Figure 2A,

160 orange) and varies with the eccentricity and SF preference of each RF. Motivated by findings of

161 psychophysical experiments that manipulated endogenous and exogenous attention (26), two SF-

162 tuned profiles are assessed-narrow and broad. The narrow profile selectively enhances a small

163 range of SFs at each eccentricity (Figure 2A, blue); the broad profile uniformly enhances SFs

\section{4 (Figure 2A, red).}


166 Suppressive drive. Suppression operates via divisive normalization $(51,52)$. Normalized responses

167 are proportional to the attention-scaled stimulus drive divided by a normalization pool plus a

168 constant $\sigma^{2}$ that increases with eccentricity. This constant adjusts the model's overall sensitivity to

169 contrast (i.e., contrast gain; Figure 2A, black). The normalization pool consists of the attention-

170 scaled stimulus drive across nearby spatial locations (surround suppression (57)), uniformly across

171 orientation (cross-orientation suppression (58)) and across preferred and neighboring SFs (cross-

172 frequency suppression (59)) of individual RFs. Such broad suppressive pools are supported by

173 physiological $(57,58,60)$ and psychophysical $(59,61,62)$ findings and models of visual processing 174 (51).

176 Spatial summation. Normalized responses are weighted and summed across space within each SF

177 and orientation filter. Spatial summation followed normalization (63), which accentuated texture-

178 defined contours within the image. The size of pooling regions scale with the SF preference of

179 each RF $(39,41)$ (Figure 2A, purple); larger for low than for high SFs. This implements an inverse

180 relation between the integration area of individual RFs and their SF tuning.

182 Target discriminability. The model generated measures of discriminability ( $\left.\mathrm{d}^{\prime}\right)$ in a texture

183 segmentation task (Figure 2B). The model generated population responses to two texture images.

184 One contained a target patch whose orientation differed from its surround (target-present) and the

185 other consisted of uniform orientation throughout (target-absent). The vector length (i.e., Euclidean

186 norm) of the difference between population responses indexed d'. This measure is proportional to

187 behavioral performance, assuming the addition of normally distributed noise after normalization. 


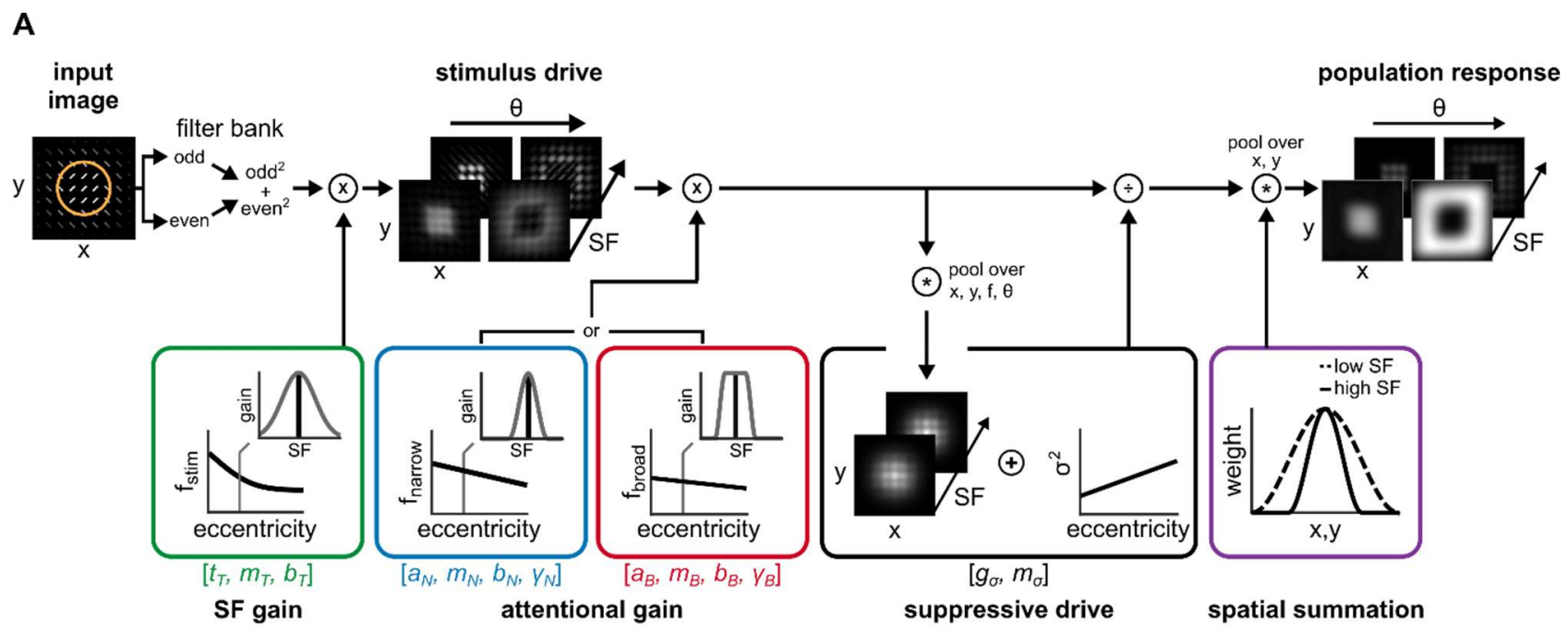

B

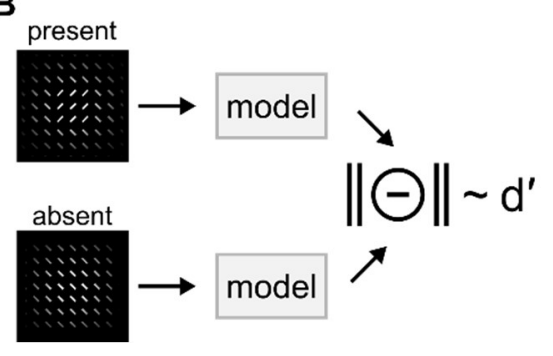

Figure 2. Image computable model of attention and spatial resolution (A) Model structure. A filter bank of linear receptive fields decomposes an image. Filter responses are squared and summed across quadrature-phase pairs (odd, even), yielding contrast energy outputs. SF gain scales contrast energy across SF and eccentricity (green box). The solid black line depicts the center frequency of the tuning function $\left(f_{\text {stim }}\right)$; insets display the full SF tuning function at a single eccentricity. The stimulus drive characterizes contrast energy at each pixel in the image, filtered through feature-selective and eccentricity-dependent receptive fields. Attentional gain multiplicatively scales the stimulus drive at a circumscribed region within the image (orange circle in left panel) and varies across SF and eccentricity. The center SF of attentional gain varies with eccentricity (solid black lines in blue and red boxes). Across SF, attentional gain follows either a narrow profile (blue box) or a broad profile (red box), each centered on a given frequency ( $f_{\text {narrow }}$ or $\mathrm{f}_{\text {broad }}$ ). The suppressive drive comprises the attention-scaled stimulus drive pooled across a local neighborhood of positions, SFs and uniformly across orientation. Contrast gain, $\sigma^{2}$, adjusts suppression magnitude across eccentricity. Spatial summation follows normalization (purple box) and generates the population response. Pooling area varies inversely with SF tuning. Variables displayed within the square brackets depict model parameters fit to behavior. (B) Target discriminability. Population responses for texture images with (present) or without (absent) a target patch are computed. The vector magnitude of their difference produces a metric proportional to d', assuming independent and identically distributed Gaussian output noise. 


\section{Texture stimuli, behavioral protocol and optimization strategy}

211 Stimuli. Model parameters were constrained by data from ten published psychophysical

212 experiments. Exogenous attention was manipulated in six (27, 29-32) (Figure 3A-F) and

213 endogenous attention in four experiments (32, 33) (Figure 3G-J). In each experiment, observers

214 distinguished a patch of one orientation embedded within a background of differing orientation at

215 several possible eccentricities.

Behavioral protocol. Performance was typically measured with a two-interval forced choice protocol

218 (Figure 3K). Observers maintained fixation at the display's center while viewing two intervals of

219 texture stimuli, one of which randomly contained a target texture. Different pre-cues at their optimal

220 timing manipulated exogenous or endogenous attention. Brief peripheral pre-cues manipulated

221 exogenous attention and appeared before both intervals, but near the upcoming target location in

222 the interval containing the target $(27-32,34)$. Symbolic pre-cues manipulated endogenous

223 attention. Pre-cues appeared near fixation and indicated the target location in the target-present

224 interval $(32,33)$. Attention effects were determined relative to a neutral condition, in which

225 observers distributed attention across all possible target locations. Behavioral performance

226 displayed the three signatures of texture segmentation: (i) The CPD emerged in the neutral

227 condition (Figure 1A). (ii) Peripheral pre-cues improved performance in the periphery and impaired

228 it at foveal locations (Figure 1B). (iii) Central, symbolic pre-cues improved performance at all

229 eccentricities (Figure 1C).

230 Optimization. To identify the computations that underlie each signature, we separately fit the model

231 to three subsets of behavioral data. First, the CPD was isolated from attentional effects by fitting to

232 the neutral condition from all ten experiments. Second, exogenous attentional effects were

233 assessed by fitting to neutral and peripheral cueing conditions from the six exogenous attention

234 experiments. Third, endogenous attentional effects were assessed by fitting to neutral and central

235 cueing conditions from the four endogenous attention experiments. The model was jointly fit to

236 each subset of data, with model parameters shared among experiments within a subset (Table S2-

237 S4). 
A

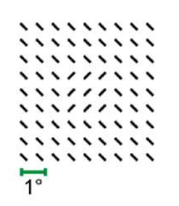

B、い、

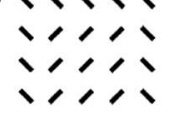

111
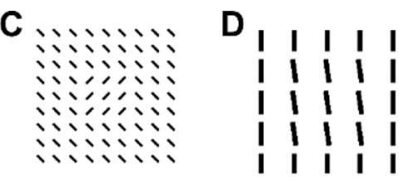

$E$

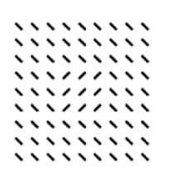

$\mathbf{F}$ 111111

111
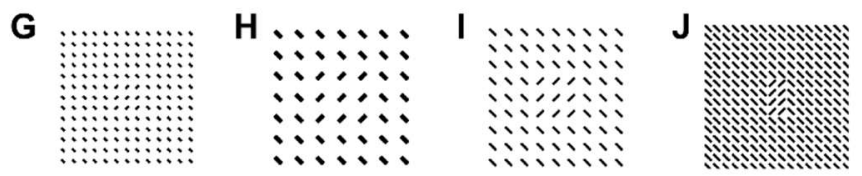

K

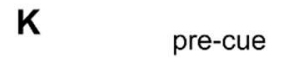

ISI

present

pre-cue
+ ISI

absent

response
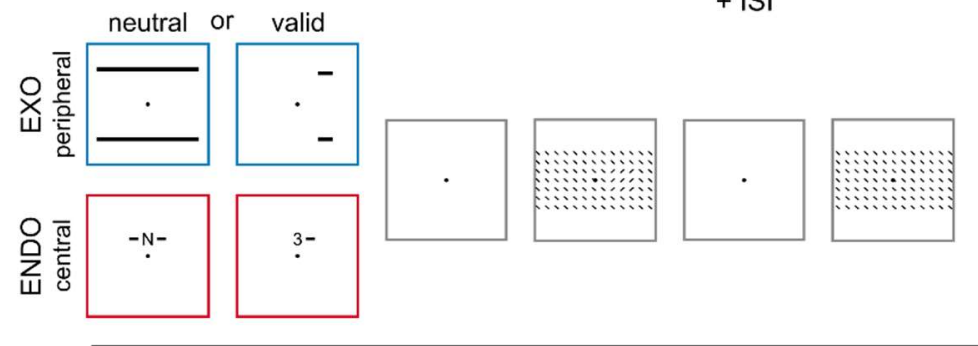

target interval?
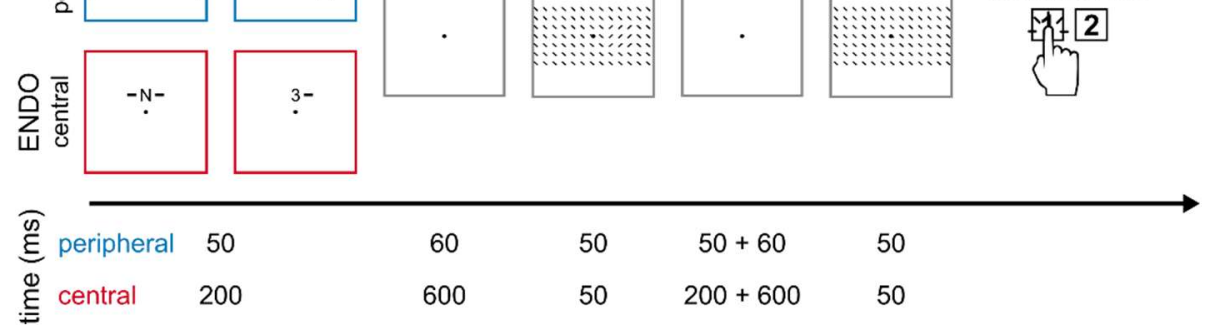

Figure 3. Texture stimuli and a typical texture segmentation behavioral protocol.

Target-present texture stimuli used in (A-F) exogenous attention and (G-J) endogenous attention experiments, displayed at their respective spatial scales. Textures displayed include:

(A) Fine and (B) coarse-scale textures used in Yeshurun \& Carrasco, 1998 (27); (C) Talgar \& Carrasco, 2002 (29) with targets placed on the vertical meridian; (D) Carrasco, Loula \& Ho, 2006 (30) wherein observers discriminated the target's orientation; (E) Yeshurun \& Carrasco, 2008 (31) where the cue's size was manipulated; (F) Experiment 2 of Yeshurun, Montagna \& Carrasco, 2008 (32) with targets placed on the horizontal meridian; (G) Experiment 1 of Yeshurun, Montagna \& Carrasco, 2008 (32) with targets placed on the horizontal meridian; (H) Experiment 3 and (I) Experiment 4 of Yeshurun, Montagna \& Carrasco, 2008 (32) wherein fine and coarse-scale textures were displayed, respectively; and (J) Barbot \& Carrasco, 2017 (33) with targets placed on 250 the intercardinal meridians.

(K) Two-interval forced choice protocol typically used to assess texture segmentation performance. EXO corresponds to exogenous attention and ENDO to endogenous attention. Numbers denote the representative timing information for each pre-cue-peripheral (blue) and central (red)_and their corresponding inter-stimulus intervals (ISI). Neutral pre-cues equally distributed attention to all possible target locations. Valid peripheral pre-cues appeared near the upcoming target location whereas valid central pre-cues symbolically indicated the upcoming target location. In the displayed example, the number " 3 " and the adjacent line indicate that the target would appear at a peripheral eccentricity in the right visual hemifield. 


\section{Contextual modulation and spatial summation mediate the CPD}

261 To identify the computations mediating the CPD, we fit the model to group-average performance

262 across all experiments' neutral condition (103 data points). 15 model parameters constrained

263 performance (Table S2). To account for differences in contrast sensitivity due to variable display

264 properties among experiments (e.g., mean luminance), foveal contrast gain ( $g_{\sigma}$; Figure 2A) was

265 independently determined for each of ten experiments (10 parameters). Two separate parameters

266 determined foveal SF preference $\left(\mathrm{t}_{\mathrm{T}}\right.$ )-one shared among exogenous attention studies and another

267 among endogenous attention studies. The remaining three parameters-SF bandwidth $\left(\mathrm{b}_{\mathrm{T}}\right)$, the

268 gradual increase in contrast gain $\left(m_{\sigma}\right)$ and the progressive shift to lower SFs with eccentricity $\left(m_{T}\right)-$ were shared among all experiments. Attentional gain was not included for these fits.

271 The model reproduced the CPD and its dependence on texture scale (Figure 4). For a fine-scale

272 texture —characterized by narrow, densely spaced lines_-performance peaked within the

273 parafovea (4 deg) and declined toward the fovea and periphery (Figure 4A). Differences between

274 target-present and target-absent stimuli were largest within the $2 \mathrm{cpd}$ filter (Figure 4A, middle).

275 This filter best differentiated the target patch from a homogenous texture; we denote its center SF

276 as $\mathrm{f}_{\text {fine. }}$ A coarser texture was best distinguished by lower SFs (1 cpd, $\mathrm{f}_{\text {coarse }}$ ), which exaggerated

277 the CPD, moving peak performance to a farther eccentricity ( 6 deg; Figure 4B). The CPD was

278 well-fit in all experiments (Figure 4C); 77\% of the variance was explained (95\% bootstrapped $\mathrm{Cl}=$

279 [70 80]), with the best-fitting regression line falling close to the unity line.

Previous models qualitatively matched the CPD through spatial summation $(46,48,49)$, but ignored the contributions of contextual modulation via normalization. To assess the contribution of each operation to behavior, we compared the full model to variants that either lacked components of the suppressive drive (cross-orientation, cross-frequency, and/or surround suppression) or spatial summation (Figure 4D). We restricted contextual modulation (-context) by separately

286 limiting the pool of orientations $(-\theta)$, SFs (-f), spatial positions (-x,y) or all simultaneously (-all) such

287 that suppressive modulations due to featural attributes and/or spatial positions outside each

288 receptive field's tuning were removed. The final variant lacked spatial summation (-sum), which

289 resulted in a population response that consisted of only normalized inputs. Removing spatial

290 summation attenuates the response to regions of similar orientation (e.g., target patch). Each

291 model was fit to behavioral performance in the neutral condition across all experiments and

292 compared using Akaike Information Criterion (AIC) (64) and Bayesian Information Criterion (BIC)

293 (65). 
Removing contextual modulation or spatial summation attenuated the CPD (Figure S1). We measured model performance relative to the full model, which yielded $\triangle \mathrm{AIC}$ and $\triangle \mathrm{BIC}$ scores; positive values represent a decrease in model performance. We use "M" and "Cl" to denote the median and $95 \%$ confidence interval of the bootstrapped distribution. Model performance fell without cross-orientation suppression ( $\triangle \mathrm{AIC}: \mathrm{M}=4.8, \mathrm{Cl}=[-0.19 .7] ; \Delta \mathrm{BIC}: \mathrm{M}=4.6, \mathrm{Cl}=[-0.2$ 9.6]), cross-frequency suppression ( $\triangle \mathrm{AIC}: \mathrm{M}=7.9, \mathrm{Cl}=[2.7$ 13.2]; $\Delta \mathrm{BIC}: \mathrm{M}=7.7, \mathrm{Cl}=[2.413 .8]$ ), surround suppression ( $\triangle \mathrm{AIC}: \mathrm{M}=5.4, \mathrm{Cl}=[0.0311 .0] ; \triangle \mathrm{BIC}: \mathrm{M}=5.4, \mathrm{Cl}=[-0.111 .5])$, and without all forms of contextual modulation ( $\triangle \mathrm{AIC}: \mathrm{M}=17.0, \mathrm{Cl}=[11.522 .1] ; \Delta \mathrm{BIC}: \mathrm{M}=16.9, \mathrm{Cl}=[11.6$ 22.4]). Without spatial summation, model performance decreased as well $(\triangle \mathrm{AIC}: \mathrm{M}=37.8, \mathrm{Cl}=[33.342 .6] ; \mathrm{BIC}$ : $M=37.8, C l=[33.142 .8])$. Thus, reliable reproduction of the CPD requires both contextual modulation and spatial summation.
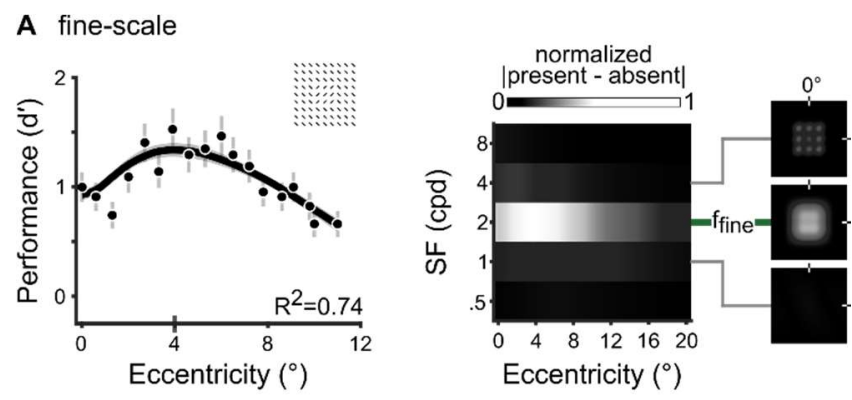

B coarse-scale
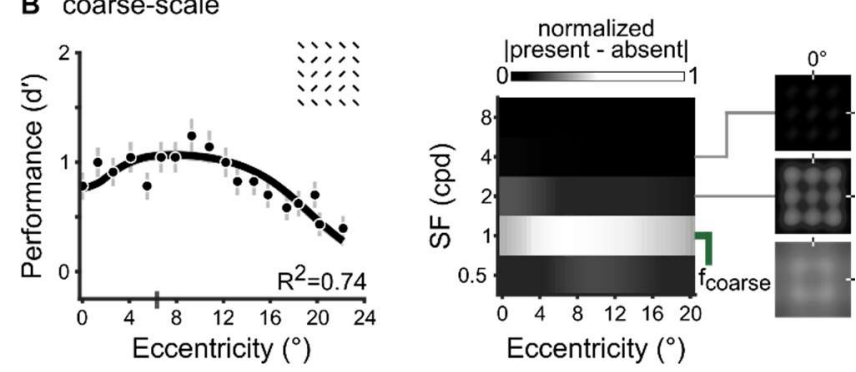

C

D
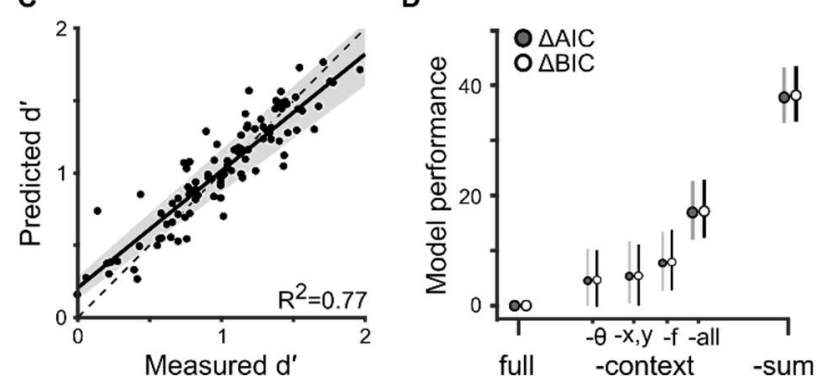

Figure 4. Contextual modulation and spatial summation mediate the CPD

(A) Left. Fit to Experiment 1 in Yeshurun \& Carrasco, 1998 (27). Dots $(n=18)$ and error bars depict group-average performance and \pm 1 SEM. The black line and shaded regions depict the median and $68 \%$ bootstrapped confidence interval of model fits. The gray vertical bar on the $x$-axis indicates the eccentricity of peak performance. The inset shows the textures stimulus. 
Middle. The matrix depicts the absolute value of differences between target-present and targetabsent population responses, normalized by the maximum across eccentricity and averaged across orientation and space. $f_{\text {fine }}$ denotes the SF filter with the largest difference between population responses. We use absolute differences only to visualize the SFs that drove discriminability.

Right. Spatial distribution of the absolute value of differences between target-present and targetabsent population responses. Each panel depicts a subset of receptive fields centered on the fovea and tuned to one of three SFs $(4,2,1 \mathrm{cpd})$ and an orientation of $30^{\circ}$.

(B) Fit to Experiment $2(n=18)$ in Yeshurun \& Carrasco, 1998 (27). The model jointly fits neutral performance with parameters shared among all ten experiments, including the data shown in $A$. Visualization follows the conventions in $A$. Note that eccentricity (x-axis) is twice that of $A . \mathrm{f}_{\text {coarse }}$ denotes the SF filter that best distinguished the coarse-scale target.

(C) Goodness-of-fit for the neutral condition across ten experiments $(n=103)$. Each dot depicts the measured (x-axis) and predicted (y-axis) performance at a given eccentricity. The solid line and shaded area depict the best-fitting regression line and its $95 \%$ confidence interval. The dashed line indicates the unity line $y=x$.

330 (D) Model comparisons using AIC and BIC. Positive values indicate models underperforming, relative to the full model. '-context' describes restrictions of contextual modulation: ' $-\theta$ ' denotes the variant without cross-orientation suppression, '-f' without cross-frequency suppression, '-x,y' without surround suppression and'-all' devoid of all contextual modulation. '-sum', denotes the model variant without spatial summation. The dots and error bars denote the median and $95 \%$ confidence interval of the bootstrap distribution.

\section{Narrow high-SF enhancement generates exogenous attention effects}

340 The model predicted behavior in neutral and peripheral cueing conditions across six experiments

341 (146 data points). Exogenous attention was modeled as a narrow SF gain profile (Figure 2, blue), 342 motivated by psychophysical measurements (26). 14 free parameters constrained model behavior

343 (Table S3). Model parameters that determined neutral cueing performance-foveal contrast gain

$344\left(g_{\sigma}\right)$, SF tuning $\left(t_{T}\right)$, SF bandwidth $\left(b_{T}\right)$, the increase in contrast gain $\left(m_{\sigma}\right)$ and the decline in SF 345 preference with eccentricity $\left(\mathrm{m}_{\mathrm{T}}\right.$ )—were configured identically as described above. Four

346 parameters, shared among experiments, determined attentional gain-foveal SF preference $\left(a_{N}\right)$,

347 the gradual shift to lower SFs with eccentricity $\left(m_{N}\right)$, SF bandwidth $\left(b_{N}\right)$ and amplitude $\left(\gamma_{N}\right)$.

348 Consequently, attention operated identically on each texture stimulus, with the spatial spread of

349 attention fixed across experiments (see Methods).

351 The model reproduced the central impairments, peripheral improvements and their variation with

352 texture scale. For a fine-scale texture, the narrow SF profile yielded improvements within the

353 parafovea $\left(4-12^{\circ}\right)$, impairments across a small range of central eccentricities $\left(0-2^{\circ}\right)$ and shifted

354 peak performance toward the periphery $\left(\sim 6^{\circ}\right.$; Figure 5A $)$. For the coarser texture, the same

355 attention profile generated improvements in the periphery (8-22 $\left.{ }^{\circ}\right)$, impairments within the

356 parafovea $\left(0-8^{\circ}\right)$ and shifted peak performance farther toward the periphery $\left(\sim 15^{\circ}\right.$; Figure $\left.5 \mathrm{~B}\right)$. 
357 A gradual shift of attentional gain toward lower SFs (26) reproduced the transition from

358 impairments to improvements across eccentricity (Figure 5C). At the fovea, attentional gain was

359 centered on a SF ( $4 \mathrm{cpd})$ higher than those distinguishing the fine- $\left(2 \mathrm{cpd}, \mathrm{f}_{\text {fine }}\right)$ or coarse-scale (1

$\left.360 \mathrm{cpd}, \mathrm{f}_{\text {coarse}}\right)$ textures. As a result, the population response shifted away from the target and impaired

361 performance. With increasing eccentricity, attentional gain progressively overlapped the SF of each

362 target, improving performance. Attention enhanced the fine-scale target SF within the parafovea

$363\left(4-12^{\circ}\right)$ then enhanced the coarse-scale target at farther eccentricities $\left(8-22^{\circ}\right)$. Overall, across the

364 six experiments, the model explained $77 \%$ of the variance $(95 \%$ bootstrapped $\mathrm{Cl}=[4982]$; Figure

365 5D).

367 Attentional gain on SFs higher than the target yielded impairments at foveal locations. This pattern

368 was consistent across all six experiments (Figure 5E). Consequently, the overlap between fine-

$369\left(f_{\text {fine }}\right)$ or coarse-scale ( $\left.f_{\text {coarse }}\right)$ targets and the SF tuning of attentional gain was minimal at the fovea

370 and peaked in the periphery (Figure $5 \mathbf{F}$ ). This mismatch between the SF tuning of attention ( $\left.f_{\text {narrow }}\right)$

371 and the target is suggested to be driven by exogenous attention operating above intrinsic SF

372 preferences at each eccentricity (26). We corroborated this relation. We compared $f_{\text {narrow }}$ to the

373 model's baseline SF tuning, indexed by the peak SF of the stimulus drive ( $f_{\text {stim, }}$ Figure $\left.2 A\right)$.

374 Consistent with empirical measurements, we found that the narrow SF profile preferred SFs higher

375 than baseline tuning (Figure S2). 

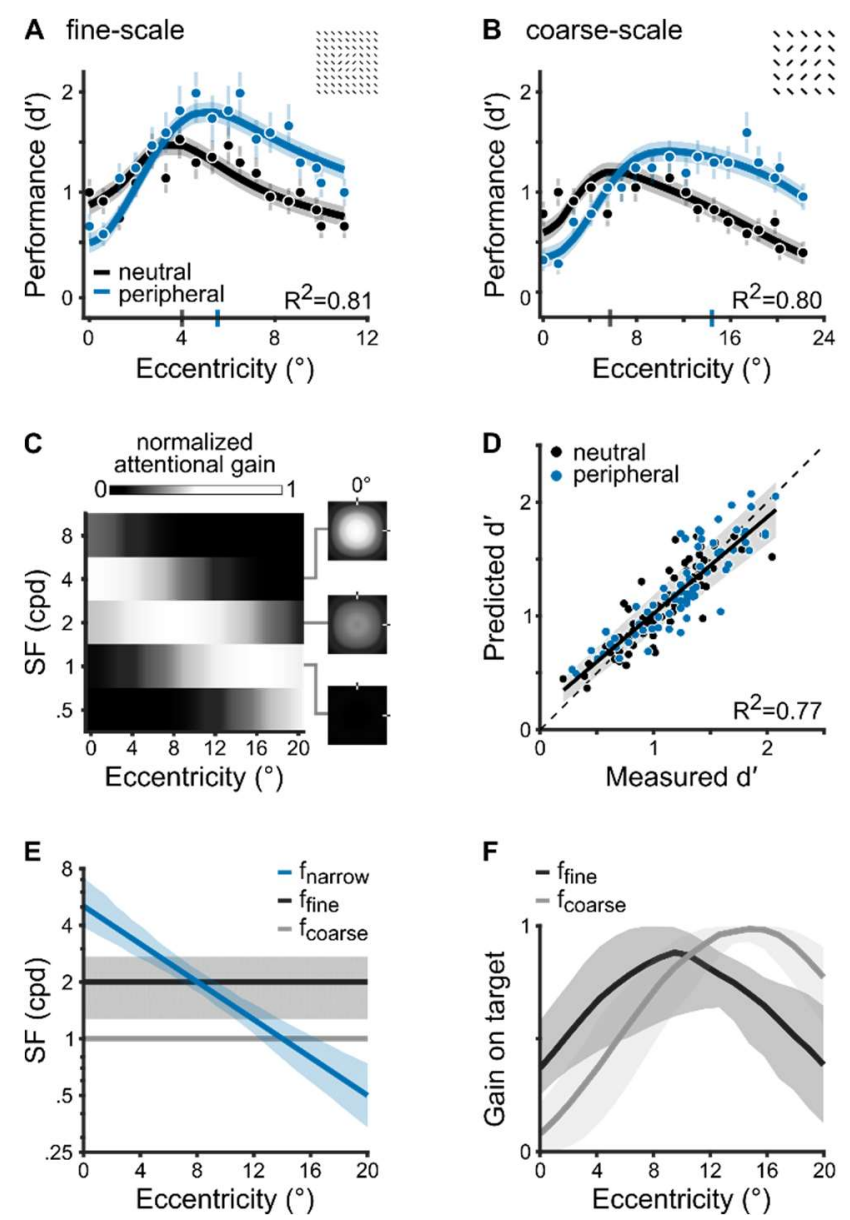

Figure 5. Narrow high-SF enhancement generates exogenous attention effects

(A) Fit to Experiment 1 in Yeshurun \& Carrasco, 1998 (27). The dots $(n=36)$ depict group-average performance and error bars denote \pm 1 SEM. The solid lines and shaded regions indicate the median and $68 \%$ confidence intervals of the bootstrapped distribution of model fits. The vertical blue bar on the $x$-axis indicates the eccentricity of peak performance with peripheral cues. (B) Fit to Experiment $2(n=36)$ in Yeshurun \& Carrasco, $1998(27)$. The model jointly fits performance on neutral and peripheral cue conditions with parameters shared among all six experiments, including the data shown in $A$. Visualization follows the conventions in $A$. (C) Best-fitting narrow gain profile. The matrix depicts attentional gain across eccentricity, normalized by the maximum and averaged across space and orientation. Matrix visualization and the panels on the right follow the conventions of Figure 4A.

(D) Goodness-of-fit for neutral and peripheral-cued performance $(n=146)$. Plotted as in Figure 4C. (E) SF preference of the narrow attentional gain profile $\left(f_{\text {narrow }}\right)$ and the SF that best distinguished fine- $\left(f_{\text {fine }}\right)$ and coarse-scale targets $\left(f_{\text {coarse }}\right)$. The solid lines and shaded areas indicate the median and $68 \%$ bootstrapped confidence interval. The shaded area for $\mathrm{f}_{\text {coase }}$ overlaps the solid line. (F) Normalized magnitude of attentional gain on the fine- and coarse-scale target SF across eccentricity (median and 68\% confidence interval of bootstrapped distribution). 
Broad SF enhancements yield endogenous attention effects

398 The model predicted group-average data from neutral and central cueing conditions across four 399 experiments (60 data points). Endogenous attention was modeled as a broad gain profile (Figure

400 2A, red) (26). 12 free parameters constrained model behavior (Table S4). Four parameters,

401 shared among experiments, determined attentional gain-foveal SF preference $\left(\mathrm{a}_{\mathrm{B}}\right)$, the decline in

402 SF preference with eccentricity $\left(m_{B}\right)$, SF bandwidth $\left(b_{B}\right)$ and amplitude $\left(\gamma_{B}\right)$.

404 The model reproduced improvements across eccentricity for both fine- (Figure 6A) and coarse-

405 scale textures (Figure 6B). To generate these improvements, attentional gain encompassed the

406 target SF for each texture scale (Figure 6C). Across all four experiments, the model explained

$40789 \%$ of the variance (95\% bootstrapped CI [67 92]; Figure 6D).

409 Endogenous attention effects were reproduced by a broad SF attentional gain that was centered 410 near the target SF across eccentricity ( $f_{\text {broad }}$ in Figure 6E). This contrasts with the narrow SF gain 411 profile that modulated higher SFs at central locations to reproduce exogenous attention effects 412 (Figure 5E). Although the center SF of attention declined with eccentricity, the modulation profile's 413 plateau ensured that it overlapped both fine- and coarse-scale target SFs across eccentricity 414 (Figure 6F). Psychophysical measurements of attentional effects on contrast sensitivity (26) 415 suggest that the SF range enhanced by endogenous attention is centered near those intrinsically 416 preferred by an observer at each eccentricity. However, our model fits to texture segmentation 417 experiments revealed that attentional gain enhanced lower SFs than baseline tuning $\left(f_{\text {stim }}\right)$ at 418 central locations (Figure S3). 
A fine-scale

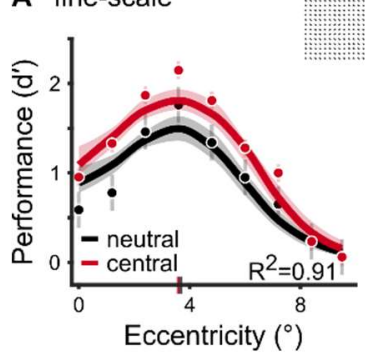

C
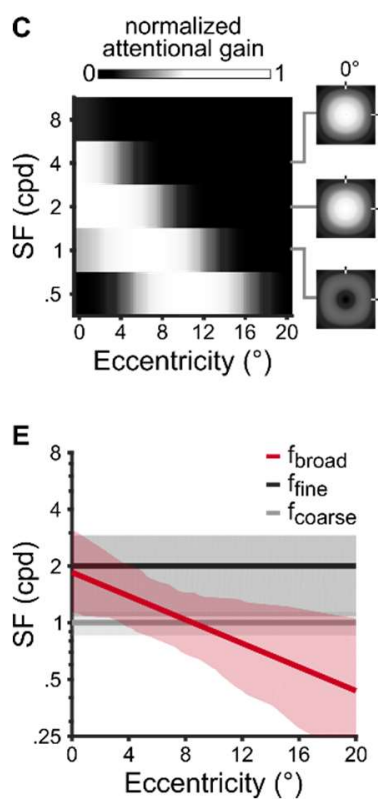
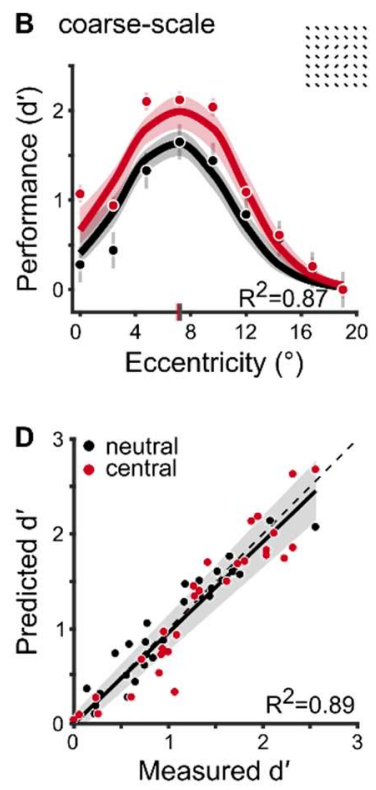

$\mathbf{F}$

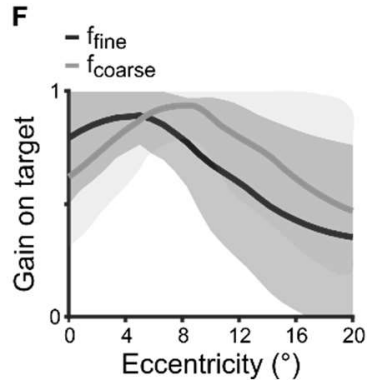

Figure 6. Broad SF enhancements yield endogenous attention effects

(A) Fit to Experiment 3 in Yeshurun, Montagna \& Carrasco, 2008 (32). The dots $(n=18)$ depict group-average performance and error bars denote \pm 1 SEM. The solid lines and shaded regions indicate the median and $68 \%$ confidence intervals of the bootstrapped distribution of model fits. The vertical red bar on the $\mathrm{x}$-axis indicates the eccentricity of peak performance with peripheral cues.

(B) Fit to Experiment $4(n=18)$ in Yeshurun, Montagna \& Carrasco, 2008 (32). The model jointly fits performance on neutral and central cue conditions with parameters shared among all four experiments, including the data shown in $A$. Visualization follows the conventions in $A$.

(C) Best-fitting broad gain profile. Plotted as in Figure 5C.

(D) Goodness-of-fit for neutral and central-cued performance $(n=60)$. Plotted as in Figure 4C.

(E) SF preference of the broad attentional gain profile $\left(f_{\text {broad }}\right)$ and the SF that best distinguished fine- $\left(f_{\text {fine }}\right)$ and coarse-scale targets $\left(f_{\text {coarse }}\right)$. The solid lines and shaded areas indicate the median and $68 \%$ bootstrapped confidence interval.

(F) Normalized magnitude of attentional gain on the fine- and coarse-scale target SF across eccentricity (median and 68\% confidence interval of bootstrapped distribution). 
440 We directly assessed whether different SF gain profiles-narrow or broad-generate the effects of

441 exogenous and endogenous attention. In addition, we compared the efficacy of SF-tuned gain

442 against a model wherein the spatial extent of attention varied across experiments while the gain

443 across SF was uniform. The spatial spread of attention is a key factor of the NMA (15), which

444 posits that its extent relative to the stimulus size helps reconcile apparent discrepancies between

445 each attention type's effects on contrast sensitivity. These predictions have been empirically tested

446 and confirmed (66). By comparing the narrow and broad SF models to the spatial extent model, we

447 directly assessed the separate contributions of SF gain and the spatial spread of attention to

448 segmentation performance (Figure 7).

450 Tuned SF gain modulation reproduced the effects of attention. The spatial extent alone was

451 insufficient to capture the effects of either exogenous ( $\triangle \mathrm{AIC}: \mathrm{M}=21.2, \mathrm{Cl}=[18.826 .0] ; \triangle \mathrm{BIC}$ :

$452 \mathrm{M}=31.7, \mathrm{Cl}=[27.934 .9]$; Figure 7A) or endogenous attention $(\Delta \mathrm{AIC}: \mathrm{M}=11.4, \mathrm{Cl}=[3.9$ 18.9]; $\triangle \mathrm{BIC}$ :

$453 \mathrm{M}=13.5, \mathrm{Cl}=\left[\begin{array}{l}5.7 \\ 20.8]\end{array}\right]$ Figure 7B). For exogenous attention, the narrow profile outperformed the

454 broad profile ( $\triangle \mathrm{AIC}: \mathrm{M}=39.1, \mathrm{Cl}=[35.5$ 42.5]; $\triangle \mathrm{BIC}: \mathrm{M}=39.1, \mathrm{Cl}=[35.9$ 42.5]; Figure 7A). For

455 endogenous attention, the broad profile outperformed the narrow profile $(\triangle \mathrm{AIC}: \mathrm{M}=25.4, \mathrm{Cl}=[17.8$

456 32.7]; $\triangle \mathrm{BIC}: \mathrm{M}=25.5, \mathrm{Cl}=[18.0$ 32.7]; Figure 7B). Decrements in model performance manifested as 457 an inability to capture impairments or improvements at eccentricities demarcating the CPD (Figure

458 S4). Thus, these model comparisons substantiate psychophysical measurements $(25,26)$ :

459 exogenous and endogenous attention effects are best explained by different attentional gain 460 profiles across SF.
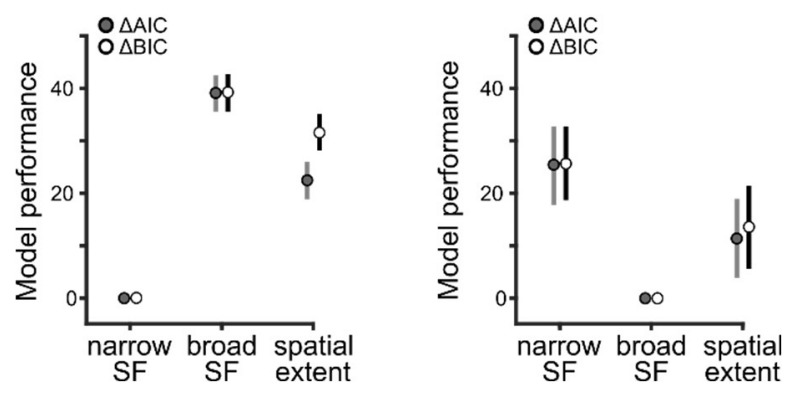

Figure 7. Different SF gain profiles govern exogenous and endogenous attention effects. $\mathrm{AIC}$ and $\mathrm{BIC}$ model comparisons for different regimes of attentional modulation for $(\mathbf{A})$ exogenous attention and (B) endogenous attention. The dots and error bars represent the median and $95 \%$ confidence intervals of the bootstrap distributions. 
Figure 8 depicts behavioral data for a variety of texture segmentation experiments. Whereas we focus on the impact of texture scale in Figure $\mathbf{5}$ and $\mathbf{6}$, the model is general. It jointly accounted for multiple target locations (vertical, Figure 8A; horizontal, Figure 8C-E; and intercardinal meridians, Figure 8F), behavioral tasks (orientation discrimination, Figure 8B) and attentional manipulations (cue size, Figure $\mathbf{8 C}$ ). Although the model was fit using texture images with fixed positions and orientations (Figure 3), it behaved similarly for textures with randomly jittered elements (Figure S5). Overall, the proposed model provides a parsimonious explanation for and a quantitative match to segmentation performance (Figure 8).
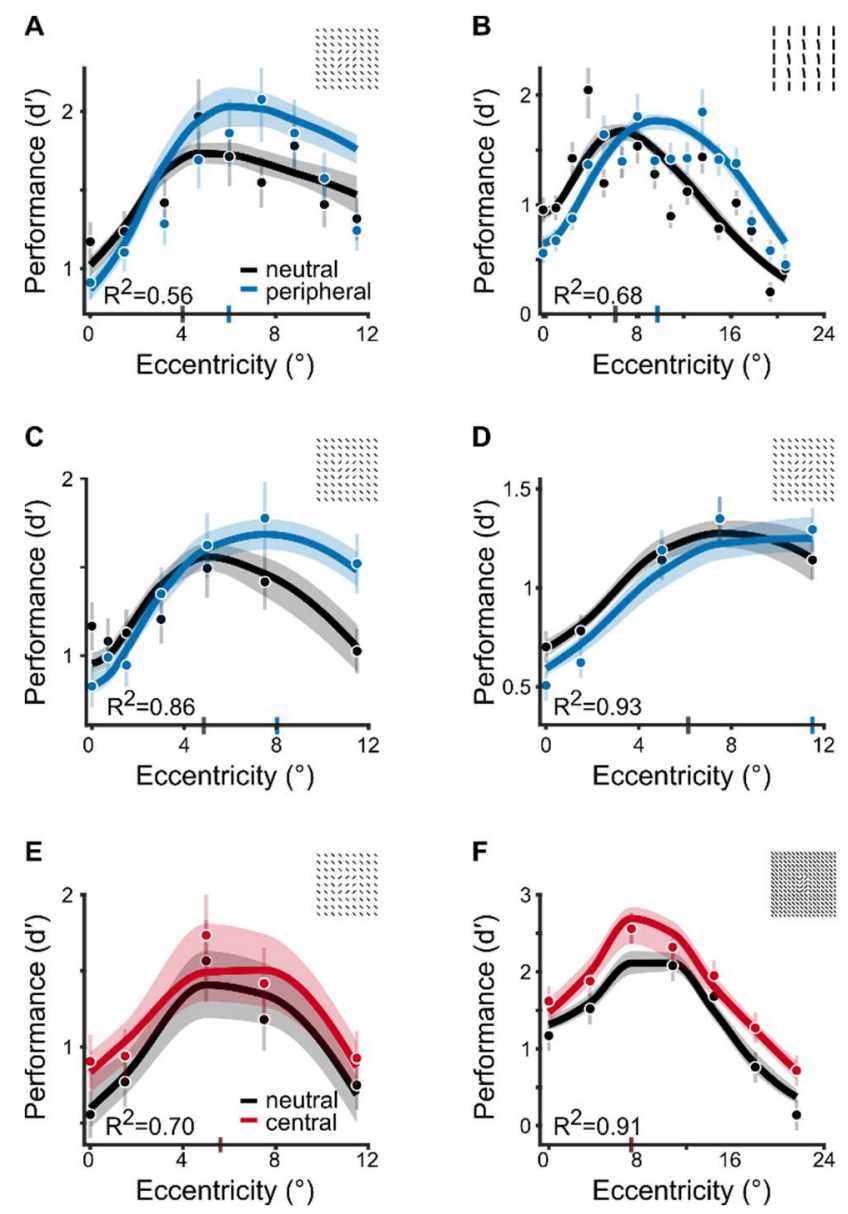

Figure 8. A parsimonious explanation for several experimental manipulations in texture segmentation

(A-D) Narrow SF gain profile fit to exogenous attention experiments. The model jointly fits these data and those displayed in Figure 5, with parameters shared among all six experiments. Insets in each panel depict the same textures displayed in Figures 3C-F, respectively. (E-F) Broad SF gain profile fit to endogenous attention experiments. The model jointly fits these data and those displayed in Figure 6, with parameters shared among all four experiments. Insets in each panel depict the same textures displayed in Figure $\mathbf{3 G}$ and $\mathbf{3} \mathbf{J}$, respectively. The dots and error bars depict group-average and \pm 1 SEM. The solid lines and shaded regions depict the median and $68 \%$ confidence intervals of the bootstrapped distribution of model fits. 


\section{Model predictions generalize to basic visual tasks}

492 To test whether this model generalizes to other basic visual tasks, we applied it to tasks mediated 493 by acuity (6) and contrast sensitivity (26), with no additional model parameters (Figure 9 ). These

494 studies separately manipulated exogenous and endogenous attention and highlight how attention

495 effects depend on the stimulus and task. In the acuity task, observers discriminated the location of

496 a small gap $\left(<1^{\circ}\right)$ in a Landolt square (Figure S6A) whereas contrast sensitivity was measured

497 with gratings in an orientation discrimination task (Figure S7A).

The model reproduced the improvements to acuity and contrast sensitivity for each attention type. On the one hand, both exogenous and endogenous attention improve acuity similarly (6). Model simulations yielded consistent visual acuity improvements for both exogenous (Figure 9A) and endogenous (Figure 9B) attention, despite different SF gain profiles underlying each attention type. On the other hand, each type of attention alters contrast sensitivity across SF differently (26).

504 Model simulations captured the differences between exogenous (Figure 9C) and endogenous

505 attention (Figure 9D). The model reproduced the narrow SF bandwidth of exogenous attention that

506 is centered on SFs higher than baseline tuning preferences (Figure S7D). It also captured the

507 broad SF modulation by endogenous attention that spanned SFs above and below baseline tuning

508 (Figure S7E). Attention effects derived from our observer model closely matched descriptive fits to

509 the data from (26) (Figure 9C-D).

511 The attention parameters were consistent across tasks (Table S6). The SF bandwidth of

512 endogenous attentional gain consistently spanned a larger range than exogenous attention (Table

513 S6, SF bw). Moreover, the rate at which SF selectivity declined with eccentricity also differed. The

514 peak SF decreased with eccentricity (Table S6, SF slope), but less so for exogenous than

515 endogenous attention, indicating that exogenous attention consistently enhanced SFs higher than

516 the peak SF of the stimulus drive (Figure S2). Lastly, we observed tradeoffs between the

517 amplitude and spatial spread of attention (Table S6). In the acuity task, the amplitude was large

$518(>8)$ and the spatial spread was narrower $\left(0.6^{\circ}\right)$ than the stimulus $\left(1^{\circ}\right)$, whereas in contrast

519 sensitivity, the amplitude was lower $(<1.5)$ and the spatial spread was broader $\left(>5^{\circ}\right)$ than the

520 stimulus $\left(4^{\circ}\right)$. Texture segmentation yielded intermediate values wherein the amplitude was $\sim 4$ for

521 a fixed spread of $4^{\circ}$. Independent of attentional effects, differences in the experimental protocol

522 and stimuli used across experiments resulted in subtle differences in the best-fitting model

523 parameters for contrast gain and the stimulus drive. Importantly, similar attention parameters

524 reproduce endogenous and exogenous attention effects in a variety of visual tasks. 
Acuity

A

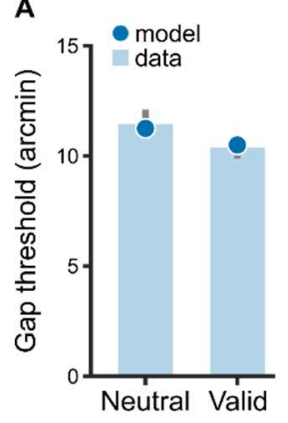

Contrast sensitivity
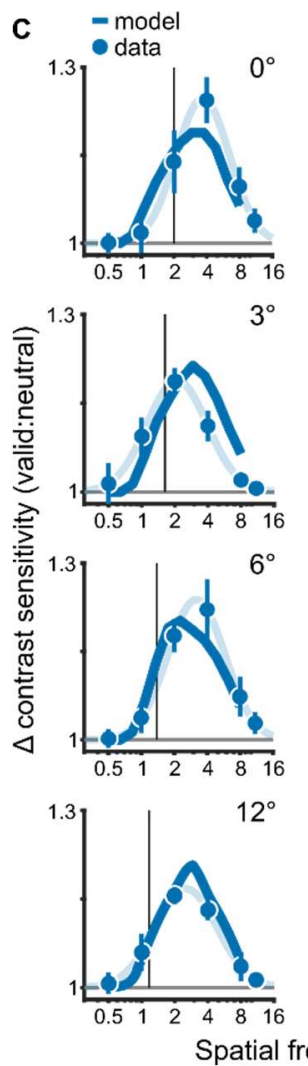

B

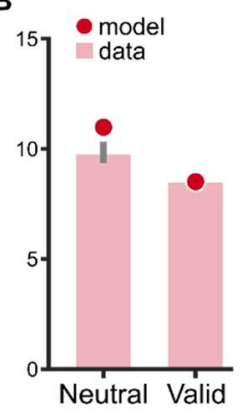

D - model
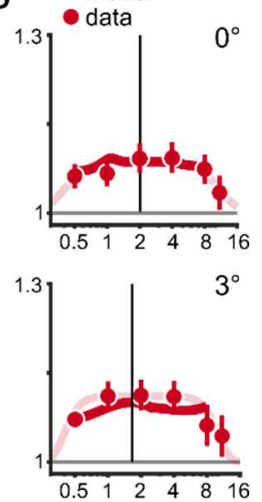

$6^{\circ}$

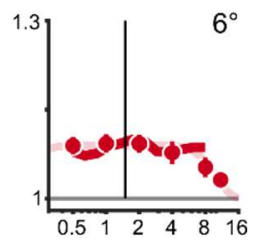

$12^{\circ}$

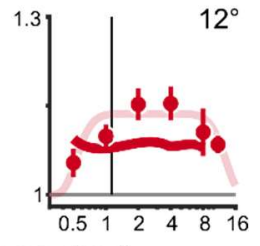

Figure 9. Model predictions generalize to other basic visual tasks. The effects of $(\mathbf{A})$ exogenous and (B) endogenous attention on gap discrimination thresholds in an acuity task. Data from (6). Lower thresholds indicate higher acuity. Bars depict group-average thresholds in neutral and valid cueing conditions. Error bars are \pm 1 SEM. Dots depict model-derived gap thresholds for the acuity task. (C) Exogenous and (D) endogenous attention effects on contrast sensitivity across SF and eccentricity, quantified as the ratio between valid and neutral contrast sensitivity. Data from (26). Values above 1 indicate an attentional enhancement of contrast sensitivity. The dots and error bars depict the group-average and \pm 1 SEM. The vertical black lines show baseline SF preferences measured in the neural condition (Figure S7). The solid colored lines show model fits to the data whereas lightly shaded lines are descriptive fits to the data from (26). In all panels, the narrow SF profile was fit to exogenous attention effects whereas the broad SF profile was fit to endogenous attention effects. 


\section{DISCUSSION}

542 We used texture segmentation as a model system to dissociate endogenous and exogenous

543 attention. To this end, we developed an image-computable model that reproduces human

544 segmentation performance and the modulations by each attention type. This model links neural

545 computations to three visual phenomena. (i) Divisive normalization and spatial summation mediate

546 the CPD (27-34, 43-46). (ii) Narrow high-SF enhancement drives exogenous attentional effects

547 (27-32, 34). (iii) Broad SF gain drives endogenous attentional modulations (32-34).

Normalization models of attention have described how spatial attention affects neural responses and behavior (e.g., $(14,15,17)$ ). Our model adopts the same algorithm specified by the ReynoldsHeeger normalization model of attention (15) (NMA) - attentional gain modulates the stimulus drive before divisive normalization. Predictions by NMA have been empirically confirmed with psychophysical experiments (66). These experiments equated seemingly distinct effects of endogenous and exogenous attention on contrast sensitivity by manipulating and accounting for

555 the spatial extent of attention.

Here, we demonstrate a critical limitation of extant models of attention. Their predictions do not extend to the differential effects on spatial resolution and do not explain the dissociation between endogenous and exogenous attention. Although the spatial extent of attention is critical for explaining effects on contrast sensitivity, our model comparisons demonstrate that it is not vital for reproducing attention effects on texture segmentation ('spatial extent' model in Figure 7 and

562 Figure S4). These results corroborate empirical evidence that manipulating the spread of attention during texture segmentation does not yield shifts between the typical effects of endogenous and exogenous attention (31).

To capture the effects of attention on texture segmentation we implemented: (i) Eccentricitydependent and SF-tuned multiplicative gains that emulate neural (54) and psychophysical (53) SF selectivity. (ii) Spatial summation, which emphasizes textural contours (39, 41, 47). (iii) Distinct SF gain profiles for endogenous and exogenous attention $(25,26)$ that scale responses prior to normalization (15), thereby adjusting the balance between fine and coarse-scale visual sensitivity. The model's distinct SF profiles instantiate a computational dissociation between each attention type that substantiates their differential impact on sensory processing. 
574 The necessity for different SF profiles is supported by empirical evidence $(25,26)$ and provides

575 insights toward the distinct roles of endogenous and exogenous attention in guiding visual

576 behavior. Previous models (e.g. $(14,15,17)$ ) demonstrate that both forms of attention improve low-

577 level visual processes that encode elementary features (e.g., contrast, orientation, motion). Here,

578 we show that attention differentially interacts with normalization to shape the competition inherent

579 in mid-level processes such as texture segmentation. Exogenous attention preferentially enhances

580 a narrow range of high SFs. Consequently, its effects prioritize fine-grained visual details at the

581 expense of competing coarse-scale features within a stimulus. In contrast, endogenous attention

582 consistently improves mid-level processing by broadly enhancing sensory encoding across fine

583 and coarse spatial scales. The computations underlying mid-level processing bridge the gap

584 between sensory encoding and object recognition (39-42). Therefore, the distinct impact by each

585 type of attention and their computational differences at this processing stage have broad

586 implications for natural visual behavior.

588 The model provides a computational framework for understanding the mechanisms underlying 589 established effects of exogenous attention on spatial resolution (27-34) (reviews (1-3)). Previous

590 studies offered qualitative descriptions that exogenous attention automatically increases spatial 591 resolution $(27-32,34)$ (reviews (1-3)) with concomitant costs in temporal resolution (22) attributed

592 to an engagement of parvocellular neurons $(22,67)$. Here, we develop an observer model that

593 anchors these qualitative descriptions onto established neural computations. In doing so, we

594 corroborate previous psychophysical experiments that found a similar high-SF preference of

595 exogenous attention $(25,26,30,68)$, specify how attentional gain changes across the visual field

596 and demonstrate its computational validity for explaining effects on perception.

598 We also provide converging evidence that exogenous attention alters perception inflexibly. By

599 comparing the model's exogenous attentional gain on textures to empirical measurements made

600 with gratings (26), we found that it consistently operates above intrinsic (i.e., baseline) SF

601 preferences despite large differences in stimuli (Figure S2). These findings suggest that in addition

602 to exogenous attentional effects being invariant to cue validity (8) and sometimes detrimental to

603 perception $(27-32,34)$, its operating range across SF is also invariant to the type of stimulus being

604 attended.

605

606 The model provides insights on the mechanisms underlying endogenous attention effects on

607 spatial resolution. Previous research has established that endogenous attention modulates texture

608 segmentation $(18,32-34,69)$ and its impact has been described as an optimization of spatial 
resolution (reviews (1-3)). We propose that a broad SF gain control mechanism yields these perceptual improvements. Our proposal complements previous reports that endogenous attention uniformly excludes noise across SF (70), but seemingly conflicts with an earlier explanation that

612 endogenous attention suppresses sensitivity to high SFs to improve texture segmentation (33).

613 However, suppressed high-SF sensitivity at foveal locations would decrease cross-frequency

614 suppression $(59,61)$ and result in an effective dominance of lower SFs, which is compatible with

615 our findings (Figure S3).

Moreover, we provide converging evidence of the flexibility of endogenous attention. We found that the model's SF preference during texture segmentation differed from those measured with gratings (26). This discrepancy suggests that the impact of endogenous attention depends on the properties of the attended stimulus and the nature of the task, consistent with the notion of a flexible endogenous attentional mechanism $(8,32-34)$.

The effects of attention depend on divisive normalization. Without normalization, we could not reliably capture the CPD, which served as the foundation of our analyses. Previous studies demonstrate that when the pool of SFs contributing to normalization is restricted, the CPD is attenuated $(30,33,44)$. However, existing models of the CPD $(46,48,49)$ relate the phenomenon solely to an increase in receptive field size with eccentricity. Our model directly links the summation area of receptive fields to their SF tuning. Consequently, the dominant summation area increases with eccentricity as SF preferences decrease. Despite implementing an increase in receptive field size, we could not capture the CPD without accounting for the surrounding context via normalization.

Additionally, we demonstrate that spatial constraints mediate the CPD independently from limitations in temporal processing across eccentricity. The proposal that the CPD may result from slow information accrual at the fovea, which yields poor performance particularly when a backward mask limits processing time $(43)$, has been criticized $(45,46,71)$. We note that our model accounts equally well for the findings of texture segmentation studies regardless of whether they contained or omitted a mask, which minimized temporal contributions to task performance (Table S5).

639 Importantly, both endogenous and exogenous attention speed information accrual (72) across the 640 visual field $(73,74)$ and across different levels of cue validity $(8)$. Thus, effects of attention on 641 temporal processing would predict similar improvements by each attention type on the CPD, a 642 prediction clearly contradicted by the modeled studies here $(27,29-33)$. 
644 The computations implemented in the model are based on the known properties of the human and

645 non-human primate visual system. The stimulus drive simulates bottom-up responses of phase-

646 invariant complex cells in V1 (56) that vary with SF and eccentricity $(53,54)$. The model's response

647 to texture is generated through pooling bottom-up inputs, consistent with the gradual emergence of

648 texture selectivity along the visual hierarchy (75-77).

650 Exogenous attentional gain in the model result in changes to texture sensitivity; however, little is

651 known about the neural underpinnings of these effects. There are sparse demonstrations of

652 exogenous attentional modulations in visuo-occipital areas and beyond (20, 21, 78-80).

653 Transcranial magnetic stimulation of early visual cortex reveals that its activity plays a key role in

654 the generation of exogenous attention effects (81). However, future studies are required to

655 determine how the SF gain modulation we report manifests in neural populations.

657 In contrast, it is established that endogenous attention modulates cortical responses (1, 2, 13, 18,

$65820,21,36-38,82,83)$. During texture segmentation tasks, endogenous attention selectively

659 enhances V1 and V4 responses to the embedded figure, suggesting that attention spreads across

660 the target object to facilitate its segmentation (18). Our model provides complementary evidence

661 that endogenous attention optimizes SF sensitivity to improve segmentation across texture scale.

662 Yet, it is unclear how neural activity generates these SF modulations. Neuroimaging $(37,38)$ and

663 electrophysiological $(13,36)$ recordings demonstrate that spatial tuning profiles are altered by

664 endogenous attention. Such changes are consistent with, but not necessary for, the modulations of

665 spatial resolution we report.

667 Few computational models have implemented possible ways in which attention alters spatial

668 resolution. Some have proposed that attention modifies how finely a spatial region is analyzed.

669 Such changes are either driven by an attention field that adjusts the spatial profile of receptive

670 fields (13) or by attracting receptive fields toward and contracting them around the attended

671 location (19). Other models suggest an attentional prioritization that selectively tunes responses for

672 a given spatial location and attenuates responses to surrounding regions $(12,16)$. However, these

673 models neither account for differences across eccentricity nor explain attentional shifts toward fine

674 or coarse spatial scales. Critically, these models do not distinguish between endogenous and

675 exogenous attention. In contrast to these previous models, we do not propose any modifications to

676 the structure of receptive fields. Instead, we attribute changes in spatial resolution to modulations

677 of SF, a fundamental dimension of early visual processing. 
679 The fact that our model operates on arbitrary images facilitates its generalization to other visual

680 stimuli and tasks. We show that the model reproduces the differential endogenous and exogenous

681 attention effects on contrast sensitivity (Figure 9C-D). Notably, the model recreates behavior in

682 visual acuity tasks where the improvements by each attention type are similar (Figure 9A-B).

683 Unlike texture segmentation, acuity tasks always benefit from heightened spatial resolution, which

684 obscures differences between these two attention types. Recent studies that compared both

685 attention types head-to-head with the same observers, stimuli and task found that they produced

686 similar behavioral effects but modulated neural activity differently in the temporo-parietal junction

687 (20) and occipital cortex (21). Our model is consistent with these findings and highlights that

688 differences in the underlying computations can yield similar perceptual effects between

689 endogenous and exogenous attention depending on the stimulus and task.

691 Future work may extend the model to other visual phenomena. For instance, it could capture the

692 differential effects by each attention type on second-order texture perception (28, 34), second-

693 order texture contrast sensitivity (24) and temporal resolution (22, 23, 67). Lastly, it is unknown

694 how interactions between both forms of attention may affect mid-level processes like texture

695 segmentation. Endogenous attention attenuates the transient effects of exogenous attention on

696 stimulus discriminability when both are deployed concurrently (84). Therefore, it is possible that

697 endogenous attentional benefits will outweigh the costs induced by exogenous attention when both

698 are deployed simultaneously during texture segmentation. Although the experimental designs of

699 the studies we have modeled cannot address this open question, our model framework may

700 facilitate predictions of the perceptual consequences when both forms of attention are deployed.

701

702 In conclusion, we reproduce signatures of texture segmentation (27-34, 43-46) and characterize

703 the contributions of attention to a process commonly considered 'preattentive' (39, 41, 42, 44-49).

704 Moreover, we reveal the neural computations that underlie how attention modifies spatial resolution

705 (1-3). Attention scales sensitivity to high and/or low SFs, adjusting the balance between fine and

706 coarse-scale spatial resolution. Exogenous attention preferentially enhances fine details whereas

707 endogenous attention uniformly enhances fine and coarse features to optimize task performance.

708 Because the model distinguishes between endogenous and exogenous attention, varies with

709 stimulus eccentricity, flexibly implements psychophysical tasks and operates on arbitrary grayscale

710 images, it provides a general-purpose tool for assessing theories of vision and attention across the

711 visual field. 
METHODS

713

\section{Model}

We developed an observer model that simulates the response of a collection of receptive fields (RFs) each narrowly tuned to spatial position $(x, y)$, orientation $(\theta)$ and SF $(f)$. Responses varied with eccentricity $(\alpha)$. The population response $(R)$ is generated by four components: the stimulus drive $(E)$, attentional gain $(A)$, suppressive drive (S and $\sigma)$, and spatial summation $(F)$, where * represents convolution:

$$
R(f, \theta, x, y)=\frac{E(f, \theta, x, y) A(f, \alpha)}{\sigma^{2}(\alpha)+S(f, \theta, x, y)} * F(f)
$$

All model parameters are given in Table $\mathbf{S 1 .}$

Stimulus drive

The stimulus drive characterizes responses of linear RFs in the absence of suppression, attention parameterized by raised-cosine functions that evenly tiled SFs, orientations and positions.

The number of SF and orientation subbands are parameters that can be flexibly chosen. We used a set of 30 subbands comprising five SF bands and six orientation bands. The size of the stimulus image and the subband bandwidth determine the total number of SF subbands. In our simulations, images were $160 \times 160$ pixels (see Stimulus generation) and SF bandwidth (i.e., full-width at halfmaximum, FWHM) was 1 octave, which allowed for five different SF subbands. The chosen bandwidth is comparable to empirical tuning curves measured in primate electrophysiological recordings (85) and human psychophysical (53) measurements. The FWHM orientation bandwidth

$738\left(60^{\circ}\right)$ is comparable to physiological tuning curves measured in primates (86). Using narrower $\left(30^{\circ}\right)$ or wider $\left(90^{\circ}\right)$ bandwidths yielded similar results supporting the same conclusions.

The pyramid includes RFs in quadrature phase. We computed a 'contrast energy' response (56),

742 (i.e., the sum of squared responses across phase) which depends on the local spectral energy at

743 each SF, orientation and position in the image. Contrast energy is fundamental to texture

744 perception models $(39,41,47)$ and we denote it as $C(f, \theta, x, y)$. 
SF gain. Human $(26,53,87)$ and non-human primate $(54)$ contrast sensitivity is narrowly tuned to was multiplied point-by-point by a SF gain function, $T$, defined by a log-parabola $(88,89)$ :

$$
T(f, \alpha)=\exp \left(-\left[\frac{\log _{2}\left(\frac{f}{\lambda_{T}(\alpha)}\right)}{b_{T}}\right]^{2}\right)
$$

where $\alpha$ denotes the eccentricity of a RF and $b_{T}$ determines the function's SF bandwidth. The preferred SF $\left(\lambda_{T}\right)$ at a given eccentricity is given by:

$$
\lambda_{T}(\alpha)=2^{t_{T}-m_{T} \alpha}+t_{\min }
$$

SF preferences converge onto a single value in the far periphery, $t_{\min }$ (87). The preferred SF at the fovea is given by $2^{t_{T}}+t_{\min }$ and progressively shifts towards $t_{\min }$ at the rate $m_{T}$. Whereas $t_{T}$ varied during simulations (see Table S1-S4), $t_{\min }$ was fixed at $0.5 \mathrm{cpd}$ because texture stimuli produced minimal contrast energy below that SF subband. Allowing $t_{\min }$ to vary yielded similar results supporting the same conclusions.

In sum, the stimulus drive $(E)$ characterizes the contrast energy responses that vary with SF and eccentricity, computed as:

Attentional gain

Attention is implemented as an attentional gain field, $A$, that multiplies the stimulus drive point-bypoint as in the Reynolds-Heeger normalization model of attention (15). Attentional gain was window functions, $w$ :

$$
w(z ; \mu, b)=\left\{\begin{array}{cc}
0.5+0.5 \cos \left(\frac{\pi[z-\mu]}{b}\right) & \vdots \cdots \\
0 & \mu-b<z>\mu+b
\end{array}\right.
$$

where $\mu$ defined its center and $b$ defined its FWHM. The units of $\mu$ and $z$ depended on the

772 dimension: for SF each variable was in units of $\log _{2}$-transformed cycles per degree and for position

773 they were in units of degrees of visual angle. The window was defined on a logarithmic axis for SF 
774 but on a linear axis for position. SF and spatial position functions were multiplied, point-by-point, to characterize the full distribution of attentional gain.

Spatial spread. Attentional gain was centered on the target location. In our simulations, the target fell along the horizontal meridian at eccentricity $\alpha_{\text {targ }}$ (see Stimulus generation). The product of two cosine functions ( $w$, equation 5 ) defined the spread of attention: one varied as a function of $x$ and another as a function of $y$, each with an identical width $b_{\text {pos. }}$. Widths did not vary across eccentricity. $A_{p o s}$ defined the spatial spread of attention:

$$
A_{\text {pos }}(x, y)=w\left(x ; \alpha_{\text {targ }}, b_{\text {pos }}\right) w\left(y ; 0, b_{\text {pos }}\right)
$$

The precise spatial spread of attention is controversial (90) and can change based on task demands $(66,91)$. Critically, it has not been explicitly manipulated during texture segmentation tasks by varying the target's spatial uncertainty. Such a protocol has been used to test predictions of the NMA and has been demonstrated to adjust the size of the attention field (66). Instead, a previous study (31) measured exogenous attention effects while manipulating the size of a peripheral pre-cue. The authors found that exogenous attention altered performance as long as the cue was the same or smaller than the target size. In our simulations, the spread of attention was fixed at a FWHM of $4^{\circ}$ (Table S1) because it encompassed the largest target size used to constrain model parameters (Table S5). As a result, the spatial extent of attention was identical across eccentricity and experiments. Similar results were observed when the spread was fixed at $2^{\circ}$ and $3^{\circ}$. However, in the model variant wherein the spatial extent could change (see Model alternatives), the FWHM of attentional spread ( $\left.b_{p o s}\right)$ was free to vary between experiments.

SF gain profile. We implemented two gain profiles: narrow and broad (26).

Narrow profile. In the narrow model $\left(A_{N}\right)$, attentional gain was bandpass across SF. Attentional gain peaked at a given SF, $\lambda_{N}$, and fell gradually toward neighboring frequencies within its bandwidth, $b_{N}$, characterized by a cosine function:

$$
A_{N}(f, \alpha)=w\left(f ; \lambda_{N}(\alpha), b_{N}\right)
$$


The center SF of attentional gain profiles ( $\lambda_{N}$ for narrow, $\lambda_{B}$ for broad) varied with eccentricity:

$$
\lambda_{N}(\alpha)=2^{a_{N}-m_{N} \alpha}
$$

808

where $a_{N}$ (or $a_{B}$ for the broad profile) defined the center frequency at the fovea, which gradually changed with eccentricity at the rate $m_{N}\left(m_{B}\right.$ for broad).

Broad profile. The broad profile $\left(A_{B}\right)$ implemented broadband attentional gain, characterized by the sum of three overlapping cosine functions:

$$
A_{B}(f, \alpha)=w_{1}+w_{2}+w_{3}
$$

where $w_{1}=w\left(f ; \lambda_{B}(\alpha), b_{B}\right), w_{2}=w\left(f ; \lambda_{B}(\alpha)-b_{B}, b_{B}\right)$, and $w_{3}=w\left(f ; \lambda_{B}(\alpha)+b_{B}, b_{B}\right)$. The FWHM of $1.5 b_{B}$.

In sum, attentional gain multiplicatively scaled the stimulus drive uniformly across orientation, but differently across SF and eccentricity given by:

$$
A(f, \alpha)=\gamma_{B} A_{p o s} A_{B}
$$

where $A_{\text {pos }}$ and $A_{B}$ (or $A_{N}$ ) were four-dimensional matrices characterizing attentional gain across position, SF and orientation. $\gamma_{B}$ (or $\gamma_{N}$ ) defined attentional amplitude. To simulate the neutral cueing condition, amplitude was set to 1 . In addition, to assess the explanatory power of the spatial spread of attention (see Model alternatives), $A_{B}$ (or $A_{N}$ ) were set to 1 and only $\gamma$ and $A_{p o s}$ varied.

\section{Suppressive drive}

831 The suppressive drive comprised contextual modulation, computed through pooling the attention-

832 scaled stimulus drive (15) across nearby positions, all orientations and neighboring SFs. This

833 pooling procedure implemented lateral interactions between RFs and was computed via

834 convolution (15). Convolution kernels were cosine window functions ( $w$, equation 5). 
837 The bandwidth of the SF kernel, $\delta_{\mathrm{f}}$, equaled 1 octave:

$$
K_{f}=\left\{\begin{array}{cc}
1 & f_{i}-\delta_{f} \leq f_{i} \leq f_{i}+\delta_{f} \\
0 & \text { otherwise }
\end{array}\right.
$$

where $f_{i}$ denotes the center SF of a subband. This kernel summed contrast energy within and \pm 1 octave around each SF subband.

The bandwidth of the orientation kernel, $\delta_{\theta}$, equaled $180^{\circ}$, which encompassed all orientation

844 subbands:

$$
K_{\theta}=\left\{\begin{array}{cc}
1 & \theta_{i}-\delta_{\theta} \leq \theta_{i} \leq \theta_{i}+\delta_{\theta} \\
0 & \text { otherwise }
\end{array}\right.
$$

where $\theta_{\mathrm{i}}$ denotes the center orientation of a steerable pyramid subband. This kernel summed contrast energy across all orientations.

Spatial position kernels were determined by multiplying two cosine windows:

$$
K_{\text {pos }}(x, y ; f)=w\left(x ; 0, \delta_{\text {pos }}\right) w\left(y ; 0, \delta_{\text {pos }}\right)
$$

One window varied across $\mathrm{x}$, another across $\mathrm{y}$ and their centers traversed across the image during convolution. The two-dimensional kernel summed to unity, which computed the average energy within the pooled area. Kernel width, $\delta_{\text {pos }}$, equaled $\frac{2}{f}$ and was inversely proportional to subband SF $f$ and yielded two-dimensional spatial kernels, $K_{\text {pos. }}$. Kernel widths were identical across eccentricity.

Contextual modulation was characterized via separable convolution:

$$
S(f, \theta, x, y)=K_{f} *\left(K_{\theta} *\left(K_{p o s} *[E(f, \theta, x, y) A(f, \alpha)]\right)\right)
$$

where * denotes convolution of the suppression kernels, $K$. Suppression magnitude was adjusted

862 across eccentricity by $\sigma^{2}$, which controlled the level of contrast at which neural responses reached

863 half-maximum and is referred to as contrast gain. Contrast gain was implemented as an

864 exponential function across eccentricity $(26,87)$ : 


$$
\sigma^{2}(\alpha)=10^{2\left(g_{\sigma}-m_{\sigma} \alpha\right)}
$$

867 where $g_{\sigma}$ and $m_{\sigma}$ are free parameters that determine contrast gain at the fovea and the rate at

868 which it varies with eccentricity, respectively.

Spatial summation

871 Following divisive normalization, responses were weighted and summed across space, within each

872 SF and orientation subband. Summation was accomplished via convolution by cosine windows, $F$, 873 computed using equation (13). The width of each filter scaled with SF: narrow (wide) regions of 874 space were pooled for high (low) SFs (39) and did not vary with eccentricity.

\section{Decision mechanism}

877 We used signal detection theory to relate population responses to behavioral performance $\left(d^{\prime}\right)$. The 878 available signal $s$ was computed as the Euclidean norm of the difference between target-present

$879\left(\boldsymbol{r}_{\mathrm{t}}\right)$ and target-absent $\left(\boldsymbol{r}_{\mathrm{n}}\right)$ neural population responses: $s=\left\|\boldsymbol{r}_{\boldsymbol{t}}-\boldsymbol{r}_{\boldsymbol{n}}\right\|$. Performance on a

880 discrimination task is proportional to the neural responses given the assumption of additive,

881 independent and identically distributed (IID) noise. An alternative model with Poisson noise and a

882 maximum-likelihood decision rule yields the same linkage between neural response and behavioral 883 performance $(92,93)$. The signal and noise magnitude $\left(\sigma_{n}\right)$ defined behavioral performance $d^{\prime}=$

$884 \frac{s}{\sigma_{n}} \cdot \sigma_{n}=\overline{r_{\text {neutral }}} / \overline{s_{\text {neutral }}}$ where $\overline{r_{\text {neutral }}}$ denotes the observed neutral performance averaged across

885 eccentricity and $\overline{s_{\text {neutral }}}$ denotes the eccentricity-average of the signal. This ratio scaled the

886 model's predicted behavioral performance to match the observed data.

\section{Model fitting}

889 Models were optimized by minimizing the residual sum of squared error between model and 890 behavioral $d$ ' using Bayesian adaptive direct search (BADS (94)). When applicable, performance 891 data for a psychophysical experiment were converted from proportion correct, $p$, to $d^{\prime}$ with the 892 assumption of no interval bias (95): $d^{\prime}=\sqrt{2} z(p)$ where $z$ denotes the inverse normal distribution.

893 Although performance on 2IFC tasks can exhibit biases between intervals (96), our conversion 894 algorithm operated uniformly across eccentricity, which preserved the performance variation (i.e., 895 the CPD) critical for the goals of this study. 


\section{Optimization strategy}

900 Central performance drop

901 We fit the model jointly to performance on the neutral condition of all 10 texture segmentation

902 experiments (103 data points). Peripheral and central cueing conditions (for exogenous and

903 endogenous attentional conditions, respectively) were excluded to isolate the CPD. 15 free

904 parameters fit all 103 data points (Table S2). Ten separate free parameters independently

905 controlled the minimum contrast gain at the fovea $\left(g_{\sigma} ;\right.$ equation 15$)$ for each of the 10 experiments.

906 Sensitivity to contrast and SF varies for stimuli placed at isoeccentric locations around the visual

907 field; it is higher at the horizontal meridian and decreases gradually towards the vertical meridian

908 (97-100). Whereas 5 out of 6 exogenous attention experiments used targets placed on the

909 horizontal meridian, 3 out of 4 endogenous attention experiments used targets presented along the

910 intercardinal meridians (Table S5). Because SF selectivity depends on stimulus polar angle, two

911 parameters separately determined the highest preferred SF ( $t_{T}$; equation 3$)$-one shared among

912 exogenous attention experiments and the other shared among endogenous attention experiments.

913 Alternatively, we could have fit separate parameters for horizontal, vertical and intercardinal

914 meridians. However, this approach would have added a third free parameter, reducing the

915 parsimony of the model. The configuration we used yielded reasonably good fits.

917 The remaining three parameters were shared among all experiments. Each controlled the

918 bandwidth ( $b_{T}$; equation 2 ) of the tuning function $T$, the gradual shift toward lower SFs with

919 eccentricity $\left(m_{T}\right.$; equation 3$)$ and the increase in contrast gain across eccentricity $\left(m_{\sigma}\right.$; equation 15$)$.

\section{Attentional modulation}

922 To generate the effects of attention, the model was fit separately to exogenous and endogenous

923 attention experiments. We jointly fit the model to neutral and valid conditions of each experiment.

925 Exogenous attention. All six exogenous attention experiments were fit jointly (146 data points) with

92614 free parameters (Table S3). Minimum contrast gain at the fovea $\left(g_{\sigma}\right.$; equation 15$)$ was

927 determined independently for each of six experiments, yielding six free parameters. The remaining

928 eight parameters were shared among all experiments. Four determined the stimulus drive: its

929 bandwidth ( $b_{T}$; equation 2$)$, the highest preferred SF at the fovea ( $t_{T}$; equation 3 ), the shift to lower

930 SFs with eccentricity $\left(m_{T}\right.$; equation 3$)$ and the slope of contrast gain across eccentricity $\left(m_{\sigma}\right.$;

931 equation 15). The remaining four controlled the narrow SF attentional gain profile, specifically its

932 bandwidth ( $b_{N}$; equation 7$)$, center SF $\left(\lambda_{N}\right.$; equation 8$)$, the shift to lower SFs with eccentricity $\left(m_{N}\right.$;

933 equation 8$)$, and its amplitude ( $\gamma_{\mathrm{N}}$; equation 10). 
935 Endogenous attention. All four endogenous attention experiments were fit jointly (60 data points)

936 with 12 free parameters (Table S4). Minimum contrast gain at the fovea $\left(g_{\sigma}\right.$; equation 15) was

937 determined independently for each experiment, which yielded four free parameters. The remaining

938 eight parameters were shared among experiments, as described above for exogenous attention.

\section{$940 \quad$ Model alternatives}

941 To assess whether contextual modulation and spatial summation are critical for the CPD, we

942 implemented five model variants. Individual components of the suppressive drive were iteratively

943 removed: cross-orientation suppression ('- $\left.\theta^{`}\right)$, cross-frequency suppression ('-f'), surround

944 suppression ('- $\mathrm{x}, \mathrm{y}$ ') and all components simultaneously ('-all'). In a separate variant, spatial

945 summation was removed ('-sum'). We fit each variant separately to neutral performance data from

946 all ten psychophysical experiments using the configuration described in Optimization strategy,

\section{Central performance drop.}

949 In the '-all' model, each RF was suppressed by its own response, simulating an extremely narrow

950 suppressive pool. Specifically, the extent of suppressive pools $\left(\delta_{\mathrm{f}}, \delta_{\theta}, \delta_{\mathrm{pos}}\right.$; equations $\left.11-13\right)$ were

951 set to 0 . As a result, the contributions of surround, cross-orientation and cross-frequency

952 suppression were absent. The other contextual modulation variants only had a single parameter

953 set to 0 (e.g., $\delta_{\mathrm{f}}$ for cross-frequency suppression). The '-sum' variant removed spatial summation

954 (i.e., $F$ in equation 1) from the model.

956 We additionally compared the efficacy of each attentional gain profile across SF-narrow or

957 broad-in generating the effects of exogenous and endogenous attention by fitting each profile to

958 exogenous and endogenous attention experiments. To assess the explanatory power of the spatial

959 extent of attention, a third model was compared in which the spatial spread of attention ( $b_{\text {pos, }}$,

960 equation 6) varied between experiments and the gain across SF was uniform. Each model fit

961 followed the configurations described in Parameter configuration, Attentional modulation.

\section{Model comparisons}

964 We compared models using AIC (64) and BIC (65). The difference in AIC/BIC values between

965 model variants indexed model performance. '- $\theta$ ', '-f', '-x,y', '-all' and '-sum' models were compared

966 to the full model. Additionally, narrow and broad SF gain profiles as well as the spatial extent

967 model were compared. 


\section{Stimulus generation}

969 Target-present and target-absent textures were re-created to match the stimulus parameters used in each psychophysical study (Table S5). For all stimuli, each pixel subtended $0.03125^{\circ}$ (i.e., 32 pixels ${ }^{\circ}$ ), roughly matching the spatial resolution of a $1280 \times 960$ monitor display placed $57 \mathrm{~cm}$ away from the observer.

974 The full texture stimulus used in each experiment typically spanned the entire display. We

975 generated $5^{\circ}$-wide square cutouts of the texture stimulus, centered on the target location. Because

976 the model implemented visual sensitivity that varied with eccentricity, but was uniform at

977 isoeccentric locations, all targets were assumed to be presented along the horizontal eccentricity

978 for simplicity (as in equation 6).

Each texture array was composed of lines oriented $135^{\circ}$. The target comprised a patch of lines that were oriented $45^{\circ}$. One study was an exception (30) because the texture array comprised vertical lines $\left(0^{\circ}\right)$ and the target patch contained lines tilted $\pm 8^{\circ}$ (Figure 3D). In this study, observers' performed an orientation discrimination task by reporting the orientation of the target presented on each trial. To simulate orientation discrimination performance, the target-present and target-absent

To avoid edge artifacts, texture stimuli were windowed by the sum of three cosine window functions (as in equation 8) centered on the target that produced a uniform plateau covering the central 3.75 deg and fell off with cosine edges. Pixel intensities in each stimulus were constrained between 0 and 1.

991

992 Textures used to fit the model were generated without spatial or orientation jittering. In additional

993 simulations, the stimuli of two representative experiments were jittered. The stimuli for Experiment

9941 in (27) were spatially jittered (0.3 deg jitter), and the stimuli in Experiment 4 in (32), were jittered

995 spatially ( 0.34 deg jitter) and in orientation ( $55^{\circ}$ bandwidth). Jitter parameters were compatible with

996 those specified in each study.

\section{Resampling procedures}

999 We obtained confidence intervals on the parameter estimates, model predictions and AIC/BIC 1000 values by bootstrapping the data and refitting the model 100 times per configuration (Optimization 1001 strategy) and for each model variant (Model alternatives). Bootstrap samples were generated by 
1002 drawing and fitting random samples from Gaussian distributions centered on group-average

1003 performance at a given eccentricity, with the SEM for each study defining the distribution's width.

1005 To generate bootstrap samples for simulations with jittered texture stimuli, the model was first fit to

1006 the data for each experiment using a non-jittered texture. Then, the model parameters were fixed

1007 and jittered stimuli were input to the model. This procedure allowed us to assess how a fixed model

1008 behaved with variable texture inputs. One hundred unique jittered stimuli were presented to the

1009 model.

1010

1011 Cross-validation procedure

1012 To characterize how the operating range of exogenous and endogenous attention varied with

1013 eccentricity, relative to baseline tuning preferences (Figure S2-S3), we fit polynomials to empirical

1014 measurements made by (26). Leave-one-subject-out cross-validation determined the best-fitting

1015 polynomial order. Specifically, the ratio, in octaves, between the peak SF of the neutral contrast

1016 sensitivity function and the preferred SF of attentional modulation were computed for individual

1017 observers. Eccentricities were aggregated between each of the two experiments conducted. The

1018 ratio for one observer was set aside, and the remaining were averaged. Zero to second-order

1019 polynomials were fit to the group-average ratio across eccentricities. The sum of squared error to

1020 the left-out data point indexed cross-validation error. This process was iterated until each

1021 observation was left-out once, resulting in 19 total iterations. The best-fitting polynomial order was

1022 defined as one that produced the lowest median cross-validation error across all iterations.

\section{Model generalizability to basic visual tasks}

1025 We applied the same observer model to behavioral data from tasks mediated by acuity (6) and 1026 contrast sensitivity (26). The model was configured identically to what is described in the Model 1027 section above and the same model parameters were fit to behavioral data using BADS (94). To

1028 simulate the Neutral condition, attentional gain was not included in the model. Narrow SF and

1029 broad SF gain profiles were used to simulate all exogenous and endogenous attentional effects,

1030 respectively.

1031

1032 Acuity

1033 The modeling strategy for the acuity task is outlined in Figure S6. Landolt squares were inputted to 1034 the model with stimulus parameters that matched those described in (6). The squares were $1^{\circ}$ 1035 wide Landolt squares with a line thickness of $0.05^{\circ}$. Images were padded with $0.5^{\circ}$ of empty space 1036 on each side to avoid edge artifacts. Model responses were computed for Landolt squares with a 
1037 small gap $\left(<1^{\circ}\right)$ on the top or bottom. The Eucledian norm of the difference between responses

1038 indexed localization performance in the task. The model was evaluated at the eccentricity tested in

1039 the experiment $\left(9.375^{\circ}\right)$ and at 10 linearly spaced gap sizes (0-30 arcmin). We characterized the

1040 full psychometric function by interpolating between gap sizes. Interpolation was used to reduce

1041 computational load; similar psychometric functions were generated when the model was evaluated

1042 at finer intervals. The available signal for discrimination was scaled so that the maximum $\mathrm{d}^{\prime}$

1043 equaled 2 and gap thresholds were quantified as the gap size needed to attain $d^{\prime}=1$. For each

1044 attention type, 10 free parameters were fit to 14 gap thresholds (7 observers $\times 2$ cueing conditions

1045 (Neutral, Valid)).

1046

1047 Contrast sensitivity

1048 The modeling strategy for the contrast sensitivity task is outlined in Figure S7. Tilted gratings

$1049\left( \pm 45^{\circ}\right)$ were inputted to the model with stimulus parameters that matched those described in (26).

1050 Gratings were windowed by a cosine function with a FWHM of $2^{\circ}$, had one of 6 SFs $(0.5,1,2,4$

1051 and $8 \mathrm{cpd})$ and were simulated at each of the four eccentricities tested $\left(0^{\circ}, 3^{\circ}, 6^{\circ}\right.$ and $\left.12^{\circ}\right)$. We

1052 omitted the highest SF tested in (26) because it fell outside the range of SF subbands (0.5-8 cpd)

1053 used to simulate texture segmentation performance. Grating images were padded with $0.5^{\circ}$ of

1054 empty space on each side to avoid edge artifacts.

1056 To simulate the signal available to an observer in the orientation discrimination task, we computed 1057 the Eucledian norm of the difference between orthogonal gratings. This procedure was repeated 1058 for each grating SF and eccentricity. Model population responses were evaluated at 7 log-spaced 1059 levels of contrast that were interpolated to characterize the full contrast response function (Figure 1060 S7C). Similar contrast response functions were produced when the model was evaluated at finer 1061 contrast steps. We scaled the available signal by the magnitude of internal noise to yield stimulus 1062 discriminability (Decision mechanism). Because internal noise varies with SF (101), the available 1063 signal was scaled such that the maximum d' at the fovea equaled 2 for each SF. Contrast 1064 thresholds were then determined as the level of contrast required to reach $d^{\prime}=1$ and their inverse 1065 indexed contrast sensitivity. For each attention type, 10 free parameters were fit to 360 contrast 1066 thresholds (9 observers $\times 2$ cueing conditions (neutral, valid) $\times 4$ eccentricities $\times 5$ SFs). 


\section{REFERENCES}

1073 1. M. Carrasco, Visual attention: The past 25 years. Vision Research 51, 1484-1525 (2011).

$10742 . \quad$ K. Anton-Erxleben, M. Carrasco, Attentional enhancement of spatial resolution: linking

1075

1076

1077

1078

1079

1080

1081

1082

1083

1084

1085

1086

1087

1088

1089

1090

1091

1092

1093

1094

1095

1096

1097

1098

1099

1100

1101

1102

1103

1104

1105

1106

1107

1108

1109

1110

1111

1112

1113

1114

1115

1116

1117

1118

1119

1120

1121 behavioural and neurophysiological evidence. Nature Reviews Neuroscience 14, 188-200 (2013).

3. M. Carrasco, A. Barbot, How attention affects spatial resolution. Cold Spring Harbor Symposia on Quantitative Biology 79, 149-160 (2014).

4. Y. Yeshurun, M. Carrasco, Spatial attention improves performance in spatial resolution tasks. Vision research 39, 293-306 (1999).

5. M. Carrasco, P. E. Williams, Y. Yeshurun, Covert attention increases spatial resolution with or without masks: Support for signal enhancement. Journal of Vision 2, 4 (2002).

6. B. Montagna, F. Pestilli, M. Carrasco, Attention trades off spatial acuity. Vision Research 49, 735-745 (2009).

7. M. Carrasco, Y. Yeshurun, The contribution of covert attention to the set-size and eccentricity effects in visual search. Journal of Experimental Psychology: Human Perception and Performance 24, 673-692 (1998).

8. A. M. Giordano, B. McEIree, M. Carrasco, On the automaticity and flexibility of covert attention: A speed-accuracy trade-off analysis. Journal of Vision 9, 30-30 (2009).

9. M. Carrasco, S. Ling, S. Read, Attention alters appearance. Nature neuroscience 7, 308313 (2004).

10. S. Ling, M. Carrasco, Transient covert attention does alter appearance: A reply to Schneider (2006). Perception \& Psychophysics 69, 1051-1058 (2007).

11. T. Liu, J. Abrams, M. Carrasco, Voluntary attention enhances contrast appearance. Psychological science 20, 354-362 (2009).

12. J. K. Tsotsos et al., Modeling visual attention via selective tuning. Artificial intelligence 78, 507-545 (1995).

13. T. Womelsdorf, K. Anton-Erxleben, S. Treue, Receptive Field Shift and Shrinkage in Macaque Middle Temporal Area through Attentional Gain Modulation. Journal of Neuroscience 28, 8934-8944 (2008).

14. G. M. Boynton, A framework for describing the effects of attention on visual responses. Vision research 49, 1129-1143 (2009).

15. J. H. Reynolds, D. J. Heeger, The Normalization Model of Attention. Neuron 61, 168-185 (2009).

16. J. K. Tsotsos, A computational perspective on visual attention (MIT Press, 2011).

17. A. M. Ni, S. Ray, J. H. Maunsell, Tuned normalization explains the size of attention modulations. Neuron 73, 803-813 (2012).

18. J. Poort et al., The Role of Attention in Figure-Ground Segregation in Areas V1 and V4 of the Visual Cortex. Neuron 75, 143-156 (2012).

19. O. Baruch, Y. Yeshurun, Attentional attraction of receptive fields can explain spatial and temporal effects of attention. Visual Cognition 22, 704-736 (2014).

20. L. Dugué, E. P. Merriam, D. J. Heeger, M. Carrasco, Specific Visual Subregions of TPJ Mediate Reorienting of Spatial Attention. Cerebral Cortex 28, 2375-2390 (2018).

21. L. Dugué, E. P. Merriam, D. J. Heeger, M. Carrasco, Differential impact of endogenous and exogenous attention on activity in human visual cortex. Scientific Reports 10, 21274 (2020).

22. Y. Yeshurun, L. Levy, Transient spatial attention degrades temporal resolution. Psychological Science 14, 225-231 (2003).

23. E. Hein, B. Rolke, R. Ulrich, Visual attention and temporal discrimination: Differential effects of automatic and voluntary cueing. Visual Cognition 13, 29-50 (2006).

24. A. Barbot, M. S. Landy, M. Carrasco, Differential effects of exogenous and endogenous attention on second-order texture contrast sensitivity. Journal of Vision 12, 6-6 (2012). 
1122 25. A. Fernandez, S. Okun, M. Carrasco, Differential effects of endogenous and exogenous

26. M. Jigo, M. Carrasco, Differential impact of exogenous and endogenous attention on the contrast sensitivity function across eccentricity. Journal of Vision 20, 11 (2020).

27. Y. Yeshurun, M. Carrasco, Attention improves or impairs visual performance by enhancing spatial resolution. Nature 396, 72 (1998).

28. Y. Yeshurun, M. Carrasco, The locus of attentional effects in texture segmentation. Nature Neuroscience 3, 622-627 (2000).

29. C. P. Talgar, M. Carrasco, Vertical meridian asymmetry in spatial resolution: Visual and attentional factors. Psychonomic Bulletin \& Review 9, 714-722 (2002).

30. M. Carrasco, F. Loula, Y.-X. Ho, How attention enhances spatial resolution: Evidence from selective adaptation to spatial frequency. Attention, Perception, \& Psychophysics 68, 10041012 (2006).

31. Y. Yeshurun, M. Carrasco, The effects of transient attention on spatial resolution and the size of the attentional cue. Perception \& Psychophysics 70, 104-113 (2008).

32. Y. Yeshurun, B. Montagna, M. Carrasco, On the flexibility of sustained attention and its effects on a texture segmentation task. Vision Research 48, 80-95 (2008).

33. A. Barbot, M. Carrasco, Attention modifies spatial resolution according to task demands. Psychological science 28, 285-296 (2017).

34. M. Jigo, M. Carrasco, Attention alters spatial resolution by modulating second-order processing. Journal of Vision 18, 2 (2018).

35. J. Moran, R. Desimone, Selective attention gates visual processing in the extrastriate cortex. Science 229, 782-784 (1985).

36. T. Womelsdorf, K. Anton-Erxleben, F. Pieper, S. Treue, Dynamic shifts of visual receptive fields in cortical area MT by spatial attention. Nature Neuroscience 9, 1156-1160 (2006).

37. J. Fischer, D. Whitney, Attention narrows position tuning of population responses in V1. Current Biology 19, 1356-1361 (2009).

38. Barrie P. Klein, Ben M. Harvey, Serge O. Dumoulin, Attraction of position preference by spatial attention throughout human visual cortex. Neuron 84, 227-237 (2014).

39. M. S. Landy, N. Graham, "Visual Perception of Texture" in The Visual Neurosciences. (MIT Press, Cambrige, MA, 2004), pp. 1106-1118.

40. P. R. Roelfsema, Cortical algorithms for perceptual grouping. Annu. Rev. Neurosci. 29, 203-227 (2006).

41. M. S. Landy, "Texture analysis and perception" in The New Visual Neurosciences, J. S. Werner, L. M. Chalupa, Eds. (MIT Press, Cambrige, MA, 2013), pp. 639-652.

42. J. D. Victor, M. M. Conte, C. F. Chubb, Textures as probes of visual processing. Annual review of vision science 3, 275-296 (2017).

43. L. Kehrer, Central performance drop on perceptual segregation tasks. Spatial vision 4, 4562 (1989).

44. K. Morikawa, Central performance drop in texture segmentation: the role of spatial and temporal factors. Vision Research 40, 3517-3526 (2000).

45. C. Potechin, R. Gurnsey, Backward masking is not required to elicit the central performance drop. Spatial vision 16, 393-406 (2003).

46. R. Gurnsey, D. Di Lenardo, C. Potechin, Backward masking and the central performance drop. Vision Research 44, 2587-2596 (2004).

47. J. R. Bergen, E. H. Adelson, Early vision and texture perception. Nature 333, 363-364 (1988).

48. L. Kehrer, The central performance drop in texture segmentation: A simulation based on a spatial filter model. Biological Cybernetics 77, 297-305 (1997).

49. L. Kehrer, C. Meinecke, A space-variant filter model of texture segregation: Parameter adjustment guided by psychophysical data. Biological Cybernetics 88, 183-200 (2003). 
1173 50. A. Thielscher, H. Neumann, A computational model to link psychophysics and cortical cell activation patterns in human texture processing. Journal of Computational Neuroscience 22, 255-282 (2007).

51. D. J. Heeger, Normalization of cell responses in cat striate cortex. Visual Neuroscience 9, 181-197 (1992).

52. M. Carandini, D. J. Heeger, Normalization as a canonical neural computation. Nature Reviews Neuroscience 10.1038/nrn3136 (2011).

53. N. V. S. Graham, Visual pattern analyzers, Oxford psychology series (Oxford University Press, New York, 1989), pp. 646.

54. R. L. DeValois, K. K. DeValois, Spatial vision, Oxford psychology series (Oxford Univ. Press, New York, ed. 2nd ed, 1990), pp. 381.

55. E. P. Simoncelli, W. T. Freeman, E. H. Adelson, D. J. Heeger, Shiftable multiscale transforms. IEEE Transactions on Information Theory 38, 587-607 (1992).

56. E. H. Adelson, J. R. Bergen, Spatiotemporal energy models for the perception of motion. Journal of the Optical Society of America A 2, 284 (1985).

57. J. R. Cavanaugh, W. Bair, J. A. Movshon, Nature and interaction of signals from the receptive field center and surround in macaque V1 neurons. Journal of Neurophysiology 88, 2530-2546 (2002).

58. G. J. Brouwer, D. J. Heeger, Cross-orientation suppression in human visual cortex. Journal of Neurophysiology 106, 2108-2119 (2011).

59. Y. Petrov, M. Carandini, S. McKee, Two distinct mechanisms of suppression in human vision. Journal of Neuroscience 25, 8704-8707 (2005).

60. N. J. Priebe, D. Ferster, Mechanisms underlying cross-orientation suppression in cat visual cortex. Nature Neuroscience 9, 552-561 (2006).

61. D. Sagi, S. Hochstein, Lateral inhibition between spatially adjacent spatial-frequency channels? Perception \& Psychophysics 37, 315-322 (1985).

62. T. S. Meese, D. J. Holmes, Spatial and temporal dependencies of cross-orientation suppression in human vision. Proc. R. Soc. B. 274, 127-136 (2007).

63. Z. M. Westrick, M. S. Landy, Pooling of first-order inputs in second-order vision. Vision Research 91, 108-117 (2013).

64. H. Akaike, A new look at the statistical model identification. IEEE Trans. Automat. Contr. 19, 716-723 (1974).

65. G. Schwarz, Estimating the dimension of a model. Annals of statistics 6, 461-464 (1978).

66. K. Herrmann, L. Montaser-Kouhsari, M. Carrasco, D. J. Heeger, When size matters: attention affects performance by contrast or response gain. Nature Neuroscience 13, 15541559 (2010).

67. Y. Yeshurun, Isoluminant stimuli and red background attenuate the effects of transient spatial attention on temporal resolution. Vision Research 44, 1375-1387 (2004).

68. N. Megna, F. Rocchi, S. Baldassi, Spatio-temporal templates of transient attention revealed by classification images. Vision Research 54, 39-48 (2012).

69. C. Casco, A. Grieco, G. Campana, M. P. Corvino, G. Caputo, Attention modulates psychophysical and electrophysiological response to visual texture segmentation in humans. Vision research 45, 2384-2396 (2005).

70. Z.-L. Lu, B. A. Dosher, Spatial attention excludes external noise without changing the spatial frequency tuning of the perceptual template. Journal of Vision 4, 10 (2004).

71. R. Gurnsey, P. Pearson, D. Day, Texture segmentation along the horizontal meridian: nonmonotonic changes in performance with eccentricity. Journal of Experimental Psychology: Human Perception and Performance 22, 738 (1996).

72. M. Carrasco, B. McElree, Covert attention accelerates the rate of visual information processing. Proceedings of the National Academy of Sciences 98, 5363-5367 (2001).

73. M. Carrasco, A. M. Giordano, B. McElree, Temporal performance fields: Visual and attentional factors. Vision research 44, 1351-1365 (2004). 
74. M. Carrasco, A. M. Giordano, B. McElree, Attention speeds processing across eccentricity: Feature and conjunction searches. Vision research 46, 2028-2040 (2006).

75. J. Larsson, M. S. Landy, D. J. Heeger, Orientation-Selective Adaptation to First- and Second-Order Patterns in Human Visual Cortex. Journal of Neurophysiology 95, 862-881 (2006).

76. Y. El-Shamayleh, J. A. Movshon, Neuronal Responses to Texture-Defined Form in Macaque Visual Area V2. Journal of Neuroscience 31, 8543-8555 (2011).

77. L. E. Hallum, M. S. Landy, D. J. Heeger, Human primary visual cortex (V1) is selective for second-order spatial frequency. Journal of Neurophysiology 105, 2121-2131 (2011).

78. T. Liu, F. Pestilli, M. Carrasco, Transient attention enhances perceptual performance and fMRI response in human visual cortex. Neuron 45, 469-477 (2005).

79. L. Busse, S. Katzner, S. Treue, Temporal dynamics of neuronal modulation during exogenous and endogenous shifts of visual attention in macaque area MT. Proceedings of the National Academy of Sciences 105, 16380-16385 (2008).

80. F. Wang, M. Chen, Y. Yan, L. Zhaoping, W. Li, Modulation of Neuronal Responses by Exogenous Attention in Macaque Primary Visual Cortex. Journal of Neuroscience 35, 13419-13429 (2015).

81. A. Fernández, M. Carrasco, Extinguishing Exogenous Attention via Transcranial Magnetic Stimulation. Current Biology 10.1016/j.cub.2020.07.068 (2020).

82. J. H. Reynolds, L. Chelazzi, Attentional modulation of visual processing. Annu. Rev. Neurosci. 27, 611-647 (2004).

83. F. Pestilli, M. Carrasco, D. J. Heeger, J. L. Gardner, Attentional enhancement via selection and pooling of early sensory responses in human visual cortex. Neuron 72, 832-846 (2011).

84. M. A. Grubb, A. L. White, D. J. Heeger, M. Carrasco, Interactions between voluntary and involuntary attention modulate the quality and temporal dynamics of visual processing. Psychonomic Bulletin \& Review 22, 437-444 (2015).

85. R. L. De Valois, D. G. Albrecht, L. G. Thorell, Spatial frequency selectivity of cells in macaque visual cortex. Vision Research 22, 545-559 (1982).

86. D. L. Ringach, R. M. Shapley, M. J. Hawken, Orientation Selectivity in Macaque V1: Diversity and Laminar Dependence. The Journal of Neuroscience 22, 5639-5651 (2002).

87. J. S. Pointer, R. F. Hess, The contrast sensitivity gradient across the human visual field: With emphasis on the low spatial frequency range. Vision research 29, 1133-1151 (1989).

88. A. B. Watson, A. J. Ahumada, A standard model for foveal detection of spatial contrast. Journal of Vision 5, 6 (2005).

89. L. A. Lesmes, Bayesian adaptive estimation of the contrast sensitivity function: The quick CSF method. Journal of Vision 10, 1-21 (2010).

90. Y. Yeshurun, The spatial distribution of attention. Current Opinion in Psychology 29, 76-81 (2019).

91. S. V. d. Stigchel et al., The limits of top-down control of visual attention. Acta Psychologica 132, 201-212 (2009).

92. M. Jazayeri, J. A. Movshon, Optimal representation of sensory information by neural populations. Nature Neuroscience 9, 690-696 (2006).

93. F. Pestilli, S. Ling, M. Carrasco, A population-coding model of attention's influence on contrast response: Estimating neural effects from psychophysical data. Vision research 49, 1144-1153 (2009).

94. L. Acerbi, W. J. Ma, Practical Bayesian optimization for model fitting with Bayesian adaptive direct search. Advances in neural information processing systems 30, 1836-1846 (2017).

95. N. A. Macmillan, C. D. Creelman, Detection theory: A user's guide (Lawrence Erlbaum Associates, Mahwah, NJ, 2005).

96. Y. Yeshurun, M. Carrasco, L. T. Maloney, Bias and sensitivity in two-interval forced choice procedures: Tests of the difference model. Vision Research 48, 1837-1851 (2008). 
bioRxiv preprint doi: https://doi.org/10.1101/2021.01.26.428173; this version posted June 9, 2021. The copyright holder for this preprint (which

was not certified by peer review) is the author/funder, who has granted bioRxiv a license to display the preprint in perpetuity. It is made available under aCC-BY-NC-ND 4.0 International license.

1276 97. M. Carrasco, C. P. Talgar, E. L. Cameron, Characterizing visual performance fields: Effects of transient covert attention, spatial frequency, eccentricity, task and set size. Spatial vision 15, 61-75 (2001).

98. E. L. Cameron, J. C. Tai, M. Carrasco, Covert attention affects the psychometric function of contrast sensitivity. Vision Research 42, 949-967 (2002).

99. M. M. Himmelberg, J. Winawer, M. Carrasco, Stimulus-dependent contrast sensitivity asymmetries around the visual field. Journal of vision 20, 18-18 (2020).

100. A. Barbot, S. Xue, M. Carrasco, Asymmetries in visual acuity around the visual field. Journal of Vision 21, 2-2 (2021).

101. D. Silvestre, A. Arleo, R. Allard, Internal noise sources limiting contrast sensitivity. Scientific Reports 8, 2596 (2018). 


\title{
1288 Supplementary Information for
}

\section{An image-computable model on how endogenous and exogenous attention differentially alter visual perception}

1296

1297

1298

1299

1300

1301

\author{
Michael Jigo ${ }^{{ }^{*}}$, David J. Heeger ${ }^{1,2} \&$ Marisa Carrasco ${ }^{1,2}$ \\ ${ }^{1}$ Center for Neural Science and ${ }^{2}$ Department of Psychology \\ New York University, New York, NY 10003
}

1302

\section{*Corresponding author information:}

Michael Jigo (michael.jigo@nyu.edu) 
bioRxiv preprint doi: https://doi.org/10.1101/2021.01.26.428173; this version posted June 9, 2021. The copyright holder for this preprint (which was not certified by peer review) is the author/funder, who has granted bioRxiv a license to display the preprint in perpetuity. It is made available under aCC-BY-NC-ND 4.0 International license.
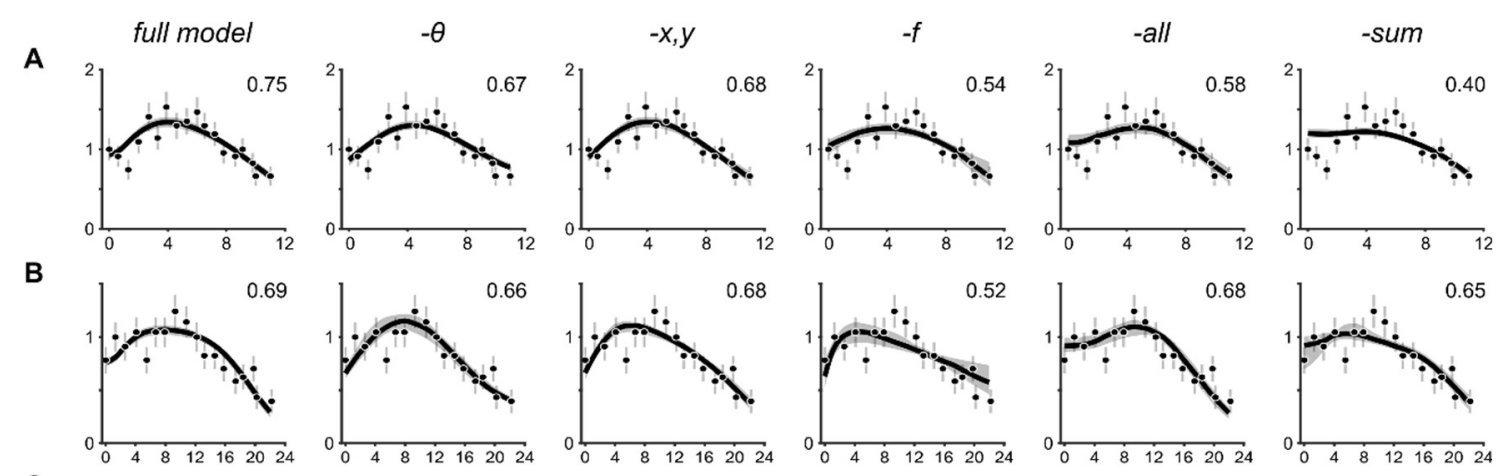

C
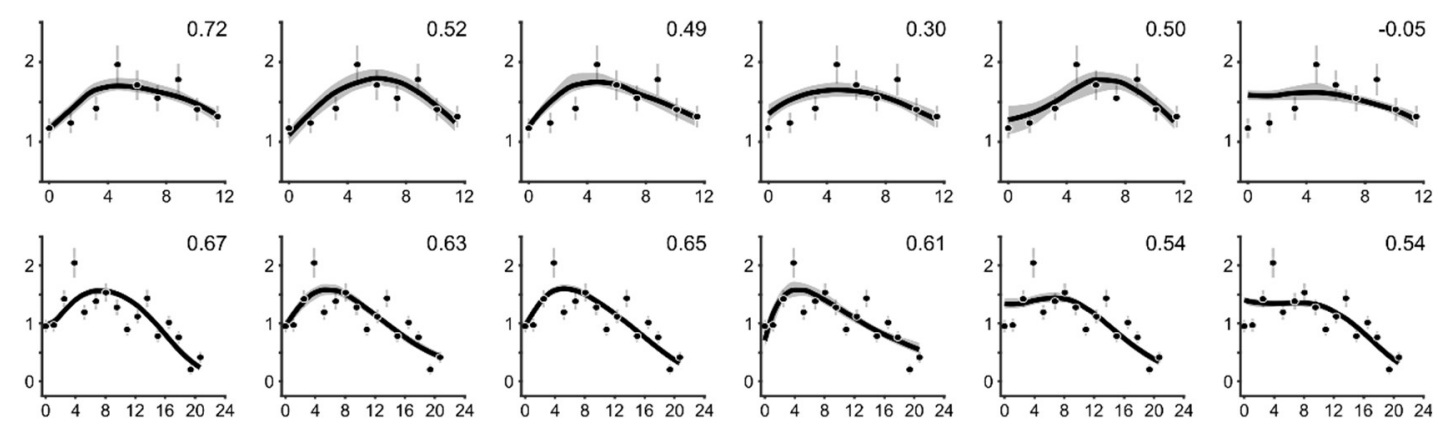

E
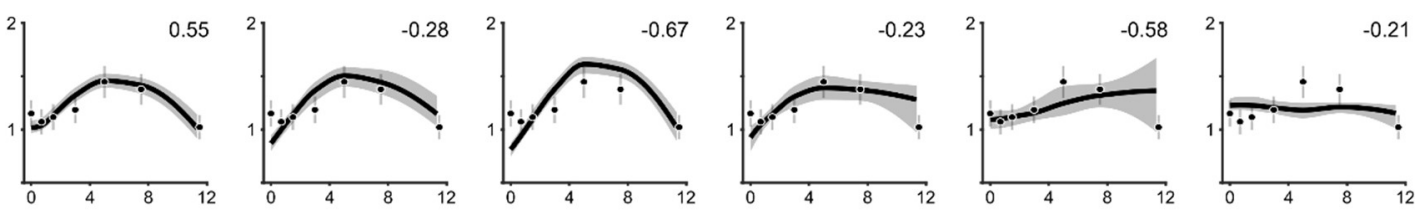

F
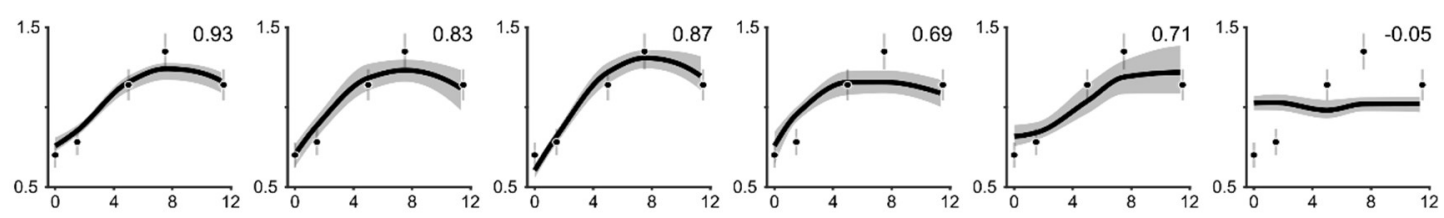

$\mathbf{G}$
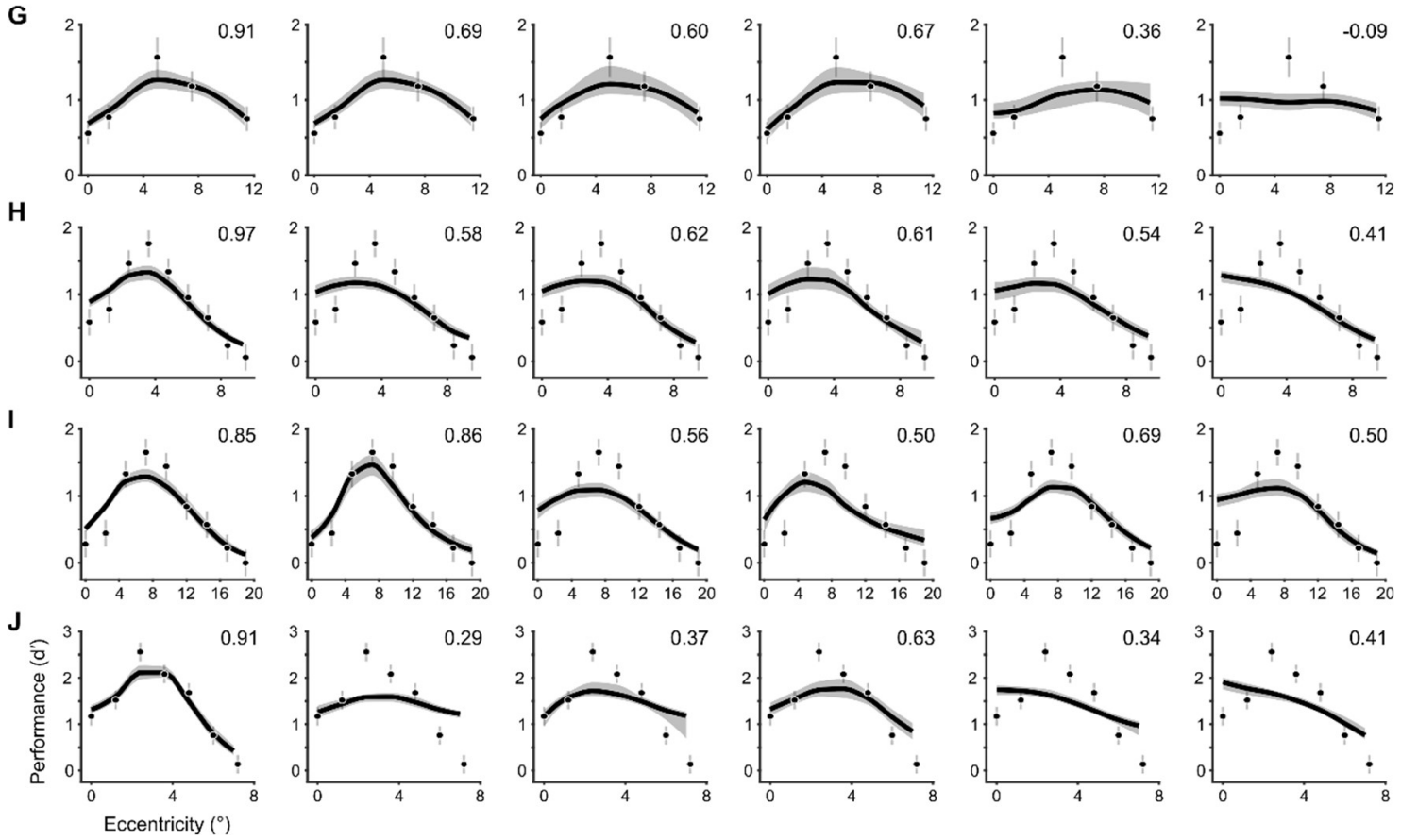
bioRxiv preprint doi: https://doi.org/10.1101/2021.01.26.428173; this version posted June 9, 2021. The copyright holder for this preprint (which was not certified by peer review) is the author/funder, who has granted bioRxiv a license to display the preprint in perpetuity. It is made available under aCC-BY-NC-ND 4.0 International license.

1306 Figure S1. Model variants fit to the neutral condition of all texture segmentation experiments. Each row depicts the behavioral data from (A) Yeshurun \& Carrasco, 1998 (27), Experiment 1; (B) Yeshurun \& Carrasco, 1998 (27), Experiment 2; (C) Talgar \& Carrasco, 2002 (29); (D) Carrasco, 1309 Loula \& Ho, 2006 (30); (E) Yeshurun \& Carrasco, 2008 (31); (F) Yeshurun, Montagna \& Carrasco, 2008 (32), Experiment 2; (G) Yeshurun, Montagna \& Carrasco, 2008 (32), Experiment 1; (H) Yeshurun, Montagna \& Carrasco, 2008 (32), Experiment 3; (I) Yeshurun, Montagna \& Carrasco, 2008 (32), Experiment 4; (J) Barbot \& Carrasco, 2017 (33). Each column shows the fit of different model variants arranged in order of best-to-worst according to the model comparisons displayed in

1314 Figure 4D: 'full' denotes the full model, '- $\theta$ ' lacks cross-orientation suppression, '- $x, y$ ' lacks

1315 surround suppression, '-f' lacks cross-frequency suppression, '-all' lacks all contextual modulation and '-sum' lacks spatial summation. Dots and error bars denote group-average performance and \pm 1 SEM. The solid lines depict the median and shaded regions depict $68 \%$ confidence intervals of the bootstrapped distribution of model fits. Values in top-right of each panel denote the median $\mathrm{R}^{2}$ of the bootstrapped distribution of model fits. Negative $R^{2}$ values indicate a model fit that captures less variance in the data than a horizontal line passing through the mean d' across eccentricity. 
A

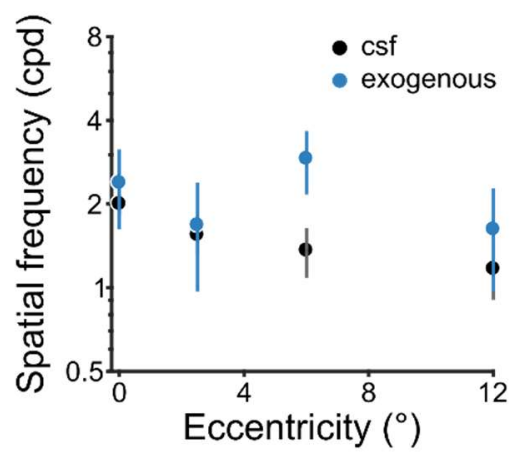

C D.
B

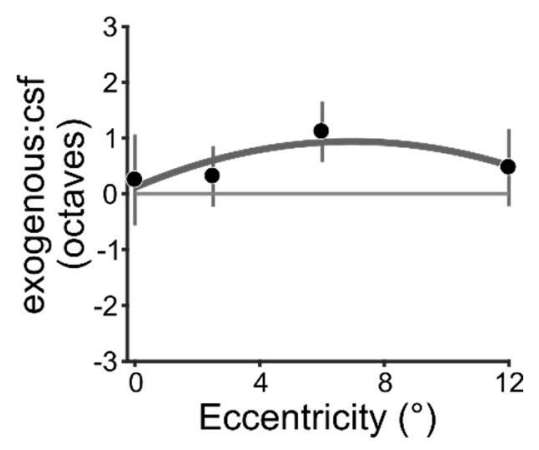

D

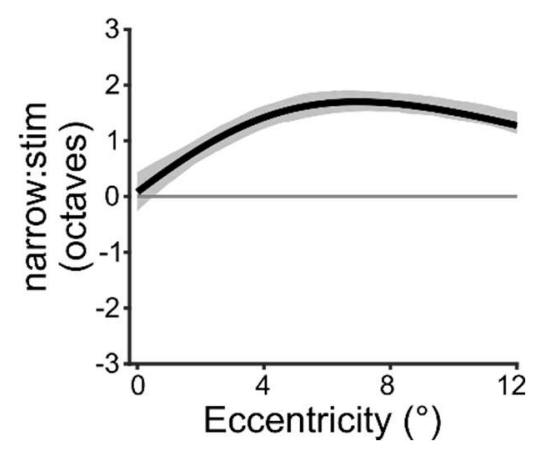

Figure S2. Spatial frequency operating range of exogenous attention.

(A) Peak spatial frequency of baseline contrast sensitivity (CSF) and exogenous attentional modulation from Jigo \& Carrasco, 2020 (26). Estimates were based on human contrast sensitivity, measured psychophysically with narrowband gratings. (B) Ratio (in octaves) of attentional and baseline peak spatial frequency tuning across eccentricity. Positive values denote an attentional preference for spatial frequencies higher than baseline. The solid line depicts the best-fitting second-order polynomial (i.e., parabola). Polynomial order was determined using leave-onesubject-out cross-validation (Methods, Cross-validation procedure). Dots in $A$ and $B$ depict group-average and error bars depict \pm 1 SEM. (C) Peak spatial frequency of the stimulus drive (stim) and the narrow SF attention gain profile (narrow). Estimates were derived from model fits to texture segmentation performance across all six exogenous attention experiments. (D) Ratio of the preferred spatial frequency for the stimulus drive and attentional gain. Solid lines indicate the median and shaded areas denotes $68 \%$ confidence interval of bootstrapped distribution in $C$ and 
A

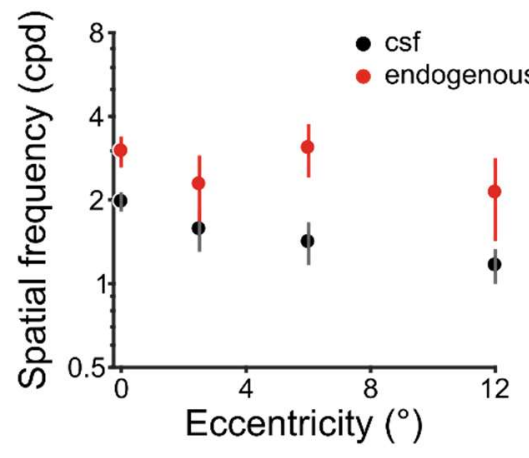

C bootstrapped distribution in $C$ and $D$.
B

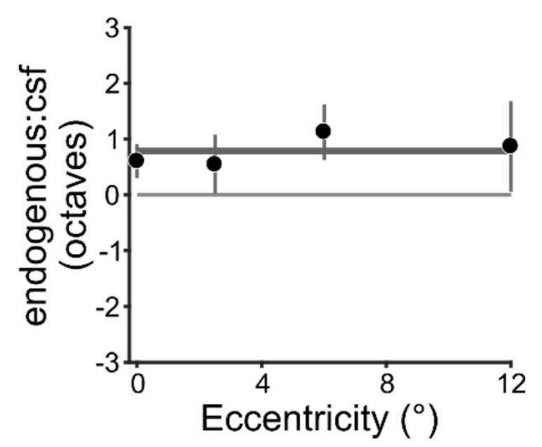

D

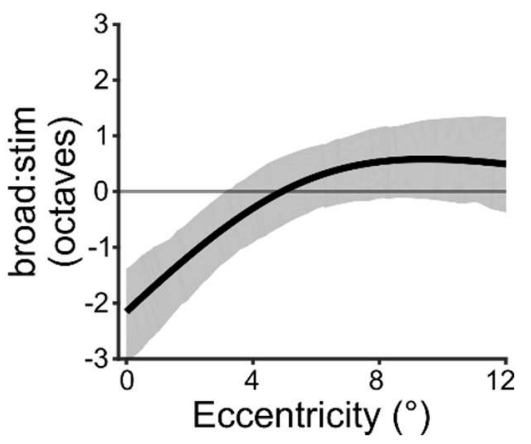

Figure S3. Spatial frequency operating range of endogenous attention.

(A) The peak spatial frequency of baseline contrast sensitivity (CSF) and the center frequency of broad endogenous attentional modulation from Jigo \& Carrasco, 2020 (26). Estimates were based on human contrast sensitivity, measured psychophysically with narrowband gratings. (B) Ratio (in octaves) of attentional and baseline and spatial frequency preferences across eccentricity. Negative values denote an attentional preference for spatial frequencies lower than baseline. The solid line depicts the best-fitting zero-order polynomial (i.e., constant). Polynomial order was determined using leave-one-subject-out cross-validation (Methods, Cross-validation procedure). Dots in $A$ and $B$ depict group-average and error bars depict \pm 1 SEM (C) The center spatial frequency of the stimulus drive (stim) and the broad attentional gain profile (broad). Estimates were derived from model fits to texture segmentation performance across all six endogenous attention experiments. (D) Ratio of the preferred spatial frequency for the stimulus drive and attentional gain. Solid lines indicate the median and shaded areas denote $68 \%$ confidence intervals of the 
bioRxiv preprint doi: https://doi.org/10.1101/2021.01.26.428173; this version posted June 9, 2021. The copyright holder for this preprint (which was not certified by peer review) is the author/funder, who has granted bioRxiv a license to display the preprint in perpetuity. It is made available under aCC-BY-NC-ND 4.0 International license.

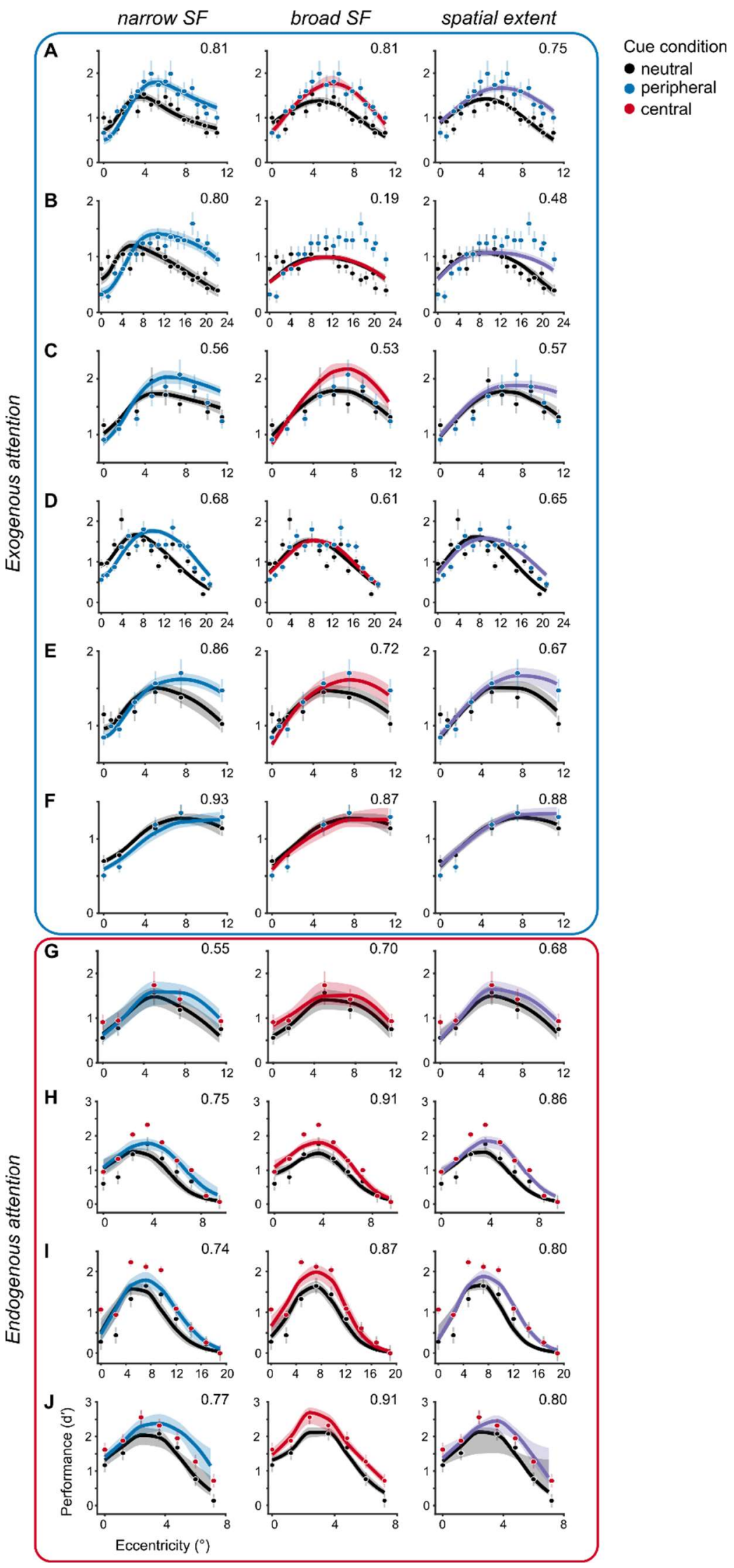


bioRxiv preprint doi: https://doi.org/10.1101/2021.01.26.428173; this version posted June 9, 2021. The copyright holder for this preprint (which was not certified by peer review) is the author/funder, who has granted bioRxiv a license to display the preprint in perpetuity. It is made available under aCC-BY-NC-ND 4.0 International license.

1355 Figure S4. Attention model variants fit to behavioral data of all ten experiments.

1356 Each row depicts a different experiment: (A) Yeshurun \& Carrasco, 1998 (27), Experiment 1; (B) Yeshurun \& Carrasco, 1998 (27), Experiment 2; (C) Talgar \& Carrasco, 2002 (29); (D) Carrasco, Loula \& Ho, 2006 (30); (E) Yeshurun \& Carrasco, 2008 (31); (F) Yeshurun, Montagna \& Carrasco, 2008 (32), Experiment 2; (G) Yeshurun, Montagna \& Carrasco, 2008 (32), Experiment 1; (H) Yeshurun, Montagna \& Carrasco, 2008 (32), Experiment 3; (I) Yeshurun, Montagna \& Carrasco, 2008 (32), Experiment 4; (J) Barbot \& Carrasco, 2017 (33). Each column depicts a different attentional gain model. The numbers in the top-right of each panel denote the median $\mathrm{R}^{2}$ of the bootstrapped distribution of model fits. 
A fine-scale

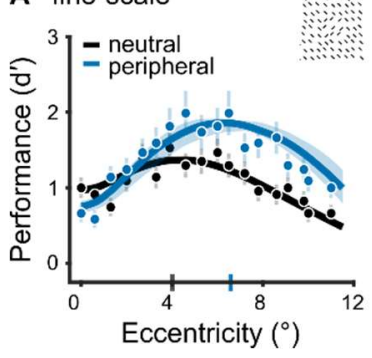

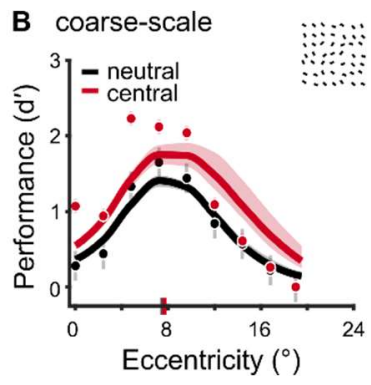

1366

1367

1368

1369

1370

1371

1372

1373

1374

1375

1376

1377

1378

1379

1380

1381

1382

1383

Figure S5. Model fits to jittered texture stimuli.

(A) Predicted performance for Experiment 1 in Yeshurun \& Carrasco, 1998 (27) using texture stimuli generated with line elements spatially jittered within the stimulus parameters of the experiment (Methods, Stimulus generation). An example jittered stimulus is shown in the topright. Solid lines indicate the median and shaded regions depict $68 \%$ confidence intervals of the bootstrap distributions of model predictions. Gray and blue ticks on $\mathrm{x}$-axis indicate peak of performance in the neutral and peripheral cueing condition, respectively. To generate the bootstrap distributions, model parameters were fixed to those that jointly captured all exogenous experiments. Then new jittered texture stimuli were input to the model on each iteration. (B) Predicted performance for Experiment 4 in Yeshurun, Montagna \& Carrasco, 2008 (32) using texture stimuli generated with line elements whose orientation and spatial location were randomly jittered within the parameters of the experiment (Methods, Stimulus generation). Solid lines indicate the median and shaded regions depict $68 \%$ confidence intervals of bootstrap distributions of model predictions. Gray and red ticks on x-axis indicate peak of performance in the neutral and central cueing condition, respectively. To generate the bootstrap distribution, model parameters were fixed to those that jointly captured all endogenous experiments, then new jittered texture stimuli were input to the model on each iteration. 
1384

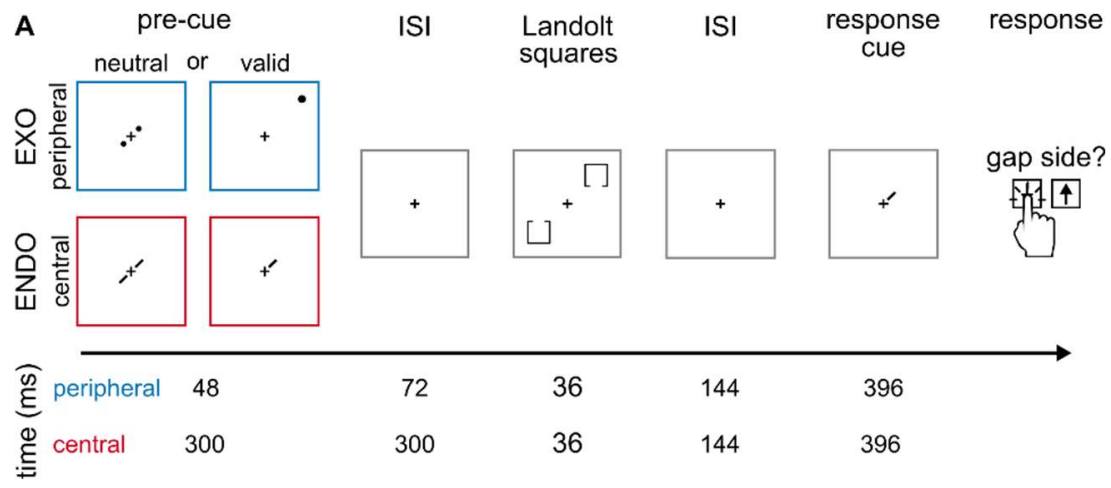

B

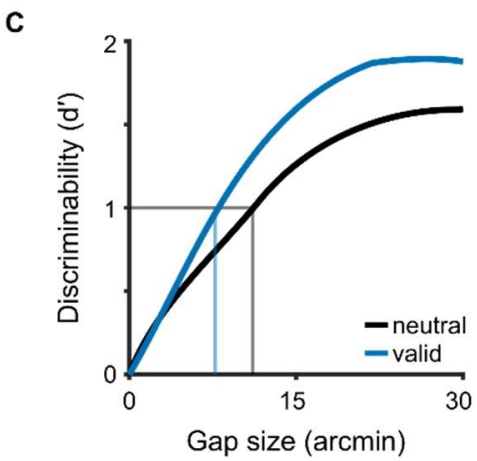

Figure S6. Behavioral protocol and modeling strategy for an acuity task.

(A) Behavioral protocol adapted from (6). Observers performed a standard Landolt acuity task. The gap size in each $1^{\circ}$-wide Landolt square varied on a trial-by-trial basis and gap thresholds were measured in conditions where attention was distributed across both target locations (neutral) or focused at a single location (valid). Peripheral cues manipulated exogenous attention (EXO) whereas central, symbolic cues manipulated endogenous attention (ENDO). On each trial, two Landolt squares appeared on one of the two main diagonals of the visual field at $9.375^{\circ}$. Observers judged whether a gap appeared at the top or bottom of the Landolt square indicated by a response cue displayed at the end of the trial. The response cue equated uncertainty of the target's location between neutral and valid cueing conditions. Gap thresholds indexed visual acuity in each condition. The timing information for peripheral (blue) and central (red) cueing conditions is given below each trial segment. (B) We modeled localization performance in this task by computing the discriminability $\left(d^{\prime}\right)$ between two Landolt squares, each with a gap at the top or bottom of the stimulus. (C) Model-derived discriminability across a range of gap sizes allowed for the creation of psychometric functions. To simulate neutral discriminability, attentional gain was not included in the model. We modeled discriminability for valid conditions with the narrow SF profile for exogenous attention and the broad SF profile for endogenous attention. Gap thresholds (vertical lines) were quantified as the gap size that resulted in $d^{\prime}=1$ (horizontal line). We fit the model's thresholds in each cueing condition to the behavioral data shown in Figure 9A-B. 
bioRxiv preprint doi: https://doi.org/10.1101/2021.01.26.428173; this version posted June 9, 2021. The copyright holder for this preprint (which was not certified by peer review) is the author/funder, who has granted bioRxiv a license to display the preprint in perpetuity. It is made available under aCC-BY-NC-ND 4.0 International license.
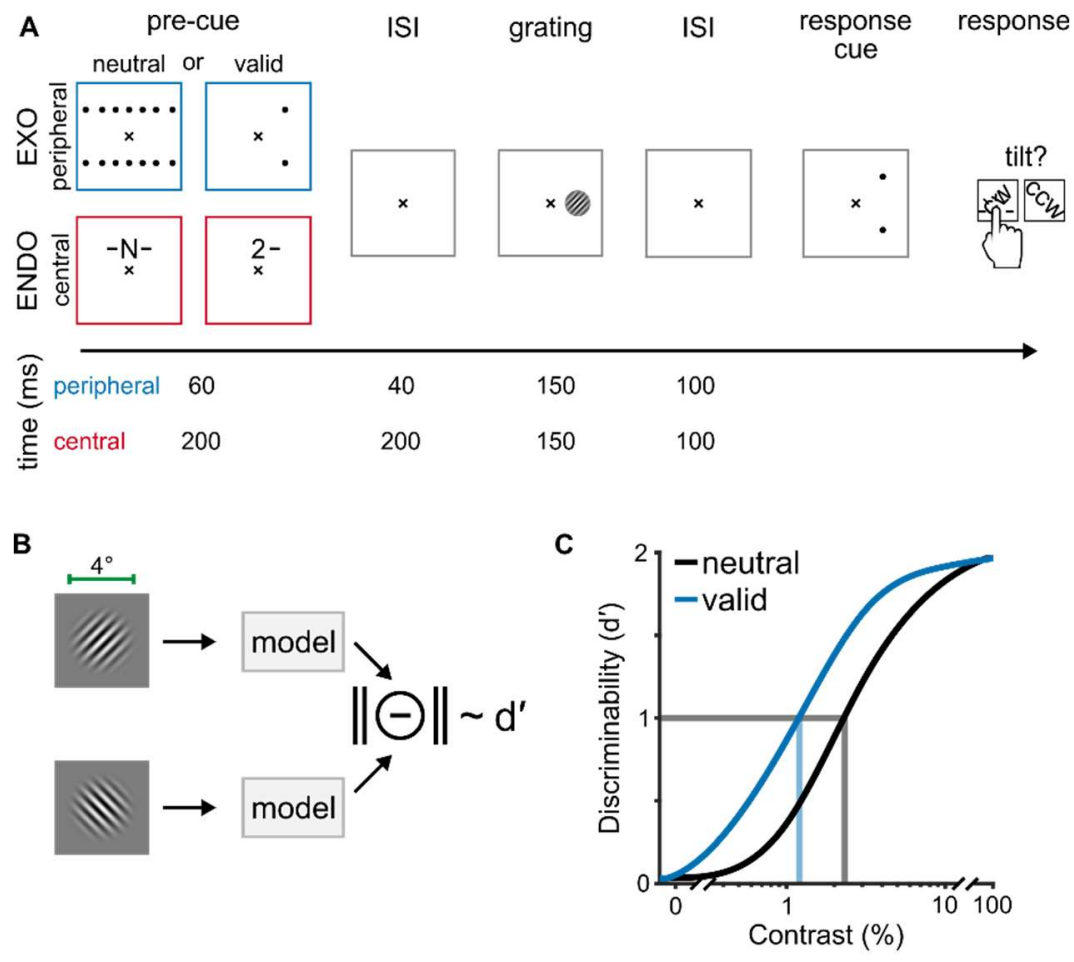

D
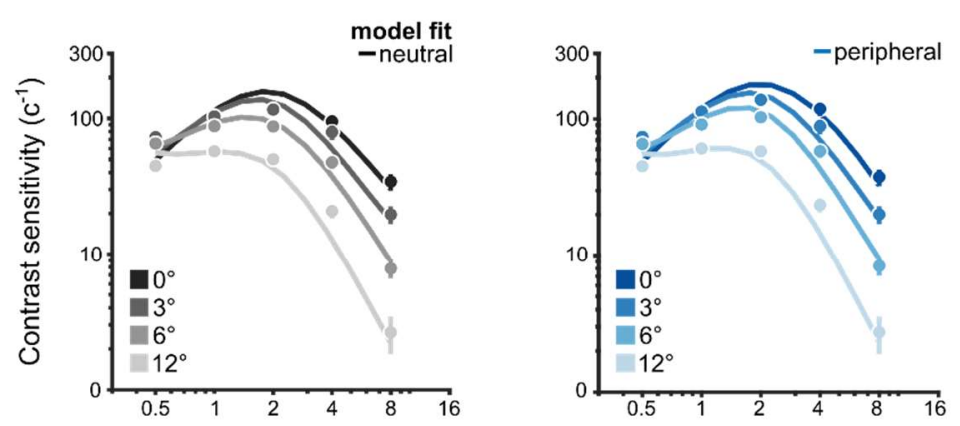

E

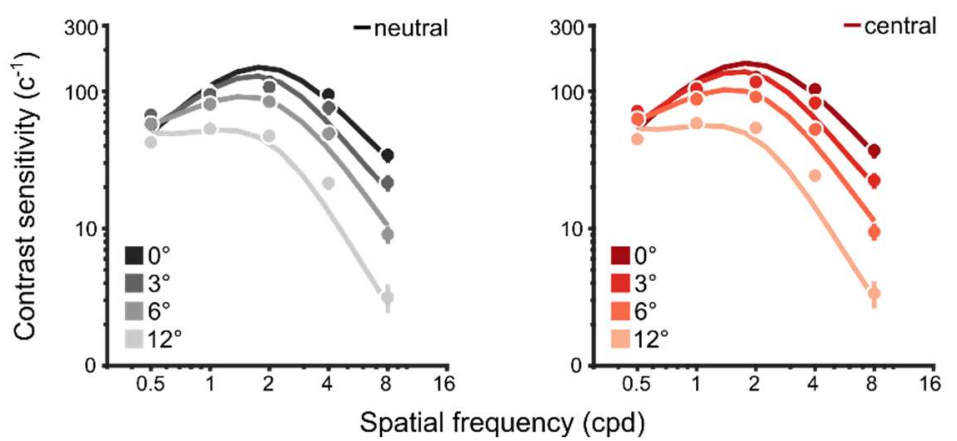

Figure S7. Behavioral protocol and modeling strategy for a contrast sensitivity task. (A) Behavioral protocol adapted from (26). Observers performed an orientation discrimination task on $4^{\circ}$-wide grating stimuli that varied in their contrast, SF and eccentricity. Grating contrast varied on a trial-bytrial basis and contrast thresholds were measured in conditions where attention was distributed across all possible target locations (neutral) or focused at a single location (valid). Peripheral cues manipulated exogenous attention (EXO) whereas central, symbolic cues manipulated endogenous attention (ENDO). On each trial, a single grating appeared along the horizontal meridian at $0^{\circ}, 3^{\circ}$, 
bioRxiv preprint doi: https://doi.org/10.1101/2021.01.26.428173; this version posted June 9, 2021. The copyright holder for this preprint (which was not certified by peer review) is the author/funder, who has granted bioRxiv a license to display the preprint in perpetuity. It is made available under aCC-BY-NC-ND 4.0 International license.

1417 cue, observers judged whether the grating was oriented clockwise (CW) or counter-clockwise (CCW) from vertical. The response cue equated uncertainty of the target's location between neutral and valid cueing conditions. The timing information for peripheral (blue) and central (red) cueing conditions is given below each trial segment. (B) We modeled orientation discrimination performance by computing the discriminability $\left(d^{\prime}\right)$ between two gratings, each tilted $\pm 45^{\circ}$ from vertical. Stimulus discriminability was simulated across a range of contrast levels for eccentricities and SFs tested in (26). (C) We simulated contrast response functions for each cueing condition. To simulate the neutral condition, attentional gain was not included in the model. Discriminability in the valid condition was modeled with the narrow SF profile for exogenous attention (i.e., peripheral cueing condition) and the broad SF profile for endogenous attention (i.e., central cueing condition). Contrast thresholds (vertical lines) were quantified as the level of contrast that resulted in $d^{\prime}=1$ (horizontal line). The inverse of contrast thresholds indexed contrast sensitivity and were fit to the behavioral data. (D) Contrast sensitivity functions for neutral (left) and peripheral cueing conditions (right). The dots and error bars depict group-average contrast sensitivity and 68\% confidence intervals for each eccentricity tested in (26). The solid lines are model fits to the behavioral data. (E) Contrast sensitivity functions for neutral (left) and central cueing conditions (right). Visualization conventions follow those in $D$. The vertical black lines in Figure 9C-D depict the peak SF of neutral contrast sensitivity functions, which indexed observers' baseline tuning preferences. The ratio between valid and neutral contrast sensitivity indexed attentional effects across SF, shown in Figure 9C-D. 
1437 Table S1. Model parameters. Bolded entries indicate model components.

\begin{tabular}{|c|c|}
\hline Parameter & Description \\
\hline \multicolumn{2}{|c|}{ Stimulus drive } \\
\hline$t_{T}$ & peak SF $\left(\log _{2}\right.$-cpd $)$ \\
\hline$t_{\min }$ & minimum preferred SF (cpd); fixed at 0.5 \\
\hline$m_{T}$ & SF tuning change across eccentricity (octaves ${ }^{\circ}$ ) \\
\hline$b_{T}$ & SF FWHM bandwidth (octaves) \\
\hline \multicolumn{2}{|c|}{ Contrast gain } \\
\hline$g_{\sigma}$ & gain at fovea \\
\hline$m_{\sigma}$ & slope along eccentricity \\
\hline \multicolumn{2}{|c|}{ Normalization pool } \\
\hline$\delta_{f}$ & SF pool bandwidth (octaves); fixed at 1 \\
\hline$\delta_{\theta}$ & Orientation pool bandwidth $\left({ }^{\circ}\right)$; fixed at 180 \\
\hline$\delta_{\text {pos }}$ & Spatial pool width $\left({ }^{\circ}\right)$; fixed at $\frac{2}{f}$ \\
\hline \multicolumn{2}{|c|}{ Spatial summation } \\
\hline$\delta_{\text {pos }}$ & Summation pool width $\left({ }^{\circ}\right)$; fixed at $\frac{2}{f}$ \\
\hline \multicolumn{2}{|c|}{ Attentional gain profile } \\
\hline$a_{N}$ or $a_{B}$ & attentional SF tuning at the fovea $\left(\log _{2}-c p d\right)$ \\
\hline$m_{N}$ or $m_{B}$ & slope of SF tuning across eccentricity (octaves $/^{\circ}$ ) \\
\hline$\gamma_{N}$ or $\gamma_{B}$ & amplitude \\
\hline$b_{N}$ or $b_{B}$ & SF FWHM bandwidth (octaves) \\
\hline$b_{\text {pos }}$ & spatial spread $\left({ }^{\circ}\right)$; fixed at FWHM of 4 \\
\hline
\end{tabular}


1439 Table S2. Free parameters for the fits to the neutral condition of all ten texture segmentation

1440 experiments. The mapping between the experiment labels $(a-j)$ and the respective references is

1441 given below the table and are consistent across all tables; italicized text describe the manipulation

1442 conducted in a given experiment. Bold values indicate the median and values within square

1443 brackets denote the $95 \% \mathrm{Cl}$ of the bootstrapped distribution of fitted parameters. min. = minimum;

1444 SF = spatial frequency; bw = bandwidth.

1445

1446

1447

1448

1449

1450

1451

1452

1453

1454

1455

1456

1457

\begin{tabular}{|c|c|c|c|c|c|c|}
\hline \multirow{3}{*}{\multicolumn{2}{|c|}{$\begin{array}{l}\text { parameter } \\
\text { description }\end{array}$}} & \multicolumn{2}{|c|}{ Contrast gain } & \multicolumn{3}{|c|}{ Stimulus drive } \\
\hline & & $g_{\sigma}$ & $m_{\sigma}$ & $t_{T}$ & $m_{T}$ & $b_{T}$ \\
\hline & & $\min$. & slope & SF peak & SF slope & SF bw \\
\hline \multirow{10}{*}{ 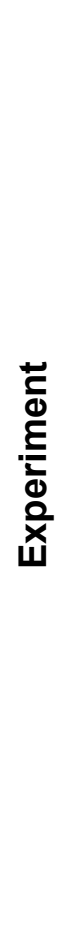 } & $a$ & $\begin{array}{c}\mathbf{2 . 3} \\
{[2.22 .5]}\end{array}$ & \multirow{10}{*}{$\begin{array}{c}\mathbf{- 0 . 0 9} \\
{[-0.09-0.08]}\end{array}$} & \multirow{6}{*}{$\begin{array}{c}1.9 \\
{[1.52 .4]}\end{array}$} & \multirow{10}{*}{$\begin{array}{c}\mathbf{- 0 . 8} \\
{[-0.9-0.6]}\end{array}$} & \multirow{10}{*}{$\begin{array}{c}1.7 \\
{[1.62 .0]}\end{array}$} \\
\hline & $b$ & $\begin{array}{c}\mathbf{2 . 3} \\
{[2.12 .5]}\end{array}$ & & & & \\
\hline & C & $\begin{array}{c}\mathbf{2 . 7} \\
{[2.52 .8]}\end{array}$ & & & & \\
\hline & d & $\begin{array}{c}\mathbf{2 . 7} \\
{[2.62 .8]}\end{array}$ & & & & \\
\hline & e & $\begin{array}{c}\mathbf{2 . 2} \\
{[2.02 .5]}\end{array}$ & & & & \\
\hline & $f$ & $\begin{array}{c}\mathbf{2 . 7} \\
{[2.42 .8]}\end{array}$ & & & & \\
\hline & g & $\left.\begin{array}{c}1.9 \\
{[1.5} \\
{[1.3]}\end{array}\right]$ & & \multirow{4}{*}{$\begin{array}{c}2.8 \\
{[2.43 .1]}\end{array}$} & & \\
\hline & $\mathrm{h}$ & $\begin{array}{c}1.8 \\
{[1.72 .0]} \\
\end{array}$ & & & & \\
\hline & $\mathrm{i}$ & $\begin{array}{c}\mathbf{2 . 6} \\
{[2.52 .8]}\end{array}$ & & & & \\
\hline & $\mathrm{j}$ & 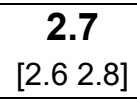 & & & & \\
\hline
\end{tabular}

a Yeshurun \& Carrasco, 1998 (27). Fine-scale texture; experiment 1.

b Yeshurun \& Carrasco, 1998 (27). Coarse-scale texture; experiment 2.

c Talgar \& Carrasco, 2002 (29). Target meridian: lower vertical.

${ }^{d}$ Carrasco, Loula \& Ho, 2006 (30). Orientation discrimination: baseline adaptation.

e Yeshurun \& Carrasco, 2008 (31). Attentional cue size: cue size 1.

f Yeshurun, Montagna \& Carrasco, 2008 (32). Target meridian: horizontal; experiment 2.

g Yeshurun, Montagna \& Carrasco, 2008 (32). Target meridian: horizontal; experiment 1

h Yeshurun, Montagna \& Carrasco, 2008 (32). Fine-scale texture; experiment 3.

'Yeshurun, Montagna \& Carrasco, 2008 (32). Coarse-scale texture; experiment 4.

jBarbot \& Carrasco, 2017 (33). Target meridian: intercardinal; baseline adaptation. 
1458 Table S3. Free parameters for the fits to neutral and peripheral cueing conditions of the six

1459 exogenous attention experiments. Bold values indicate the median and values in square brackets

1460 depict the $95 \% \mathrm{Cl}$ of the bootstrapped distribution of fitted parameters. min. = minimum; bw =

1461 bandwidth; amp. = amplitude.

1462

\begin{tabular}{|c|c|c|c|c|c|c|c|c|c|c|}
\hline \multirow{3}{*}{\multicolumn{2}{|c|}{$\begin{array}{l}\text { parameter } \\
\text { description }\end{array}$}} & \multicolumn{2}{|c|}{ Contrast gain } & \multicolumn{3}{|c|}{ Stimulus drive } & \multicolumn{4}{|c|}{ Narrow gain profile } \\
\hline & & $g_{\sigma}$ & $m_{\sigma}$ & $t_{T}$ & $\mathrm{~m}_{\mathrm{T}}$ & $\mathrm{b}_{\mathrm{T}}$ & $a_{N}$ & $\mathrm{~m}_{\mathrm{N}}$ & $b_{N}$ & $\gamma_{N}$ \\
\hline & & $\min$. & slope & SF peak & SF slope & SF bw & SF peak & SF slope & SF bw & amp. \\
\hline \multirow{6}{*}{ 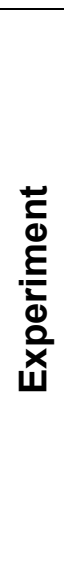 } & a & $\begin{array}{c}1.9 \\
{\left[\begin{array}{ll}1.7 & 2.2\end{array}\right]}\end{array}$ & \multirow{6}{*}{$\begin{array}{c}-\mathbf{0 . 0 5} \\
{[-0.07-0.04]}\end{array}$} & \multirow{6}{*}{$\begin{array}{c}2.1 \\
{[1.82 .8]}\end{array}$} & \multirow{6}{*}{$\begin{array}{c}-\mathbf{0 . 7} \\
{[-0.9-0.5]}\end{array}$} & \multirow{6}{*}{$\begin{array}{c}1.6 \\
{[1.42 .1]}\end{array}$} & \multirow{6}{*}{$\begin{array}{c}\mathbf{2 . 3} \\
{[1.83 .0]}\end{array}$} & \multirow{6}{*}{$\begin{array}{c}-\mathbf{0 . 2} \\
{[-0.3-0.01]}\end{array}$} & \multirow{6}{*}{$\begin{array}{c}\mathbf{2 . 9} \\
{[1.54 .8]}\end{array}$} & \multirow{6}{*}{$\begin{array}{c}\mathbf{4 . 3} \\
{[3.07 .8]}\end{array}$} \\
\hline & $b$ & $\begin{array}{c}\mathbf{1 . 7} \\
{[1.52 .1]}\end{array}$ & & & & & & & & \\
\hline & C & $\begin{array}{c}\mathbf{2 . 6} \\
{[2.22 .8]}\end{array}$ & & & & & & & & \\
\hline & $d$ & $\begin{array}{c}\mathbf{2 . 1} \\
{[2.82 .5]}\end{array}$ & & & & & & & & \\
\hline & e & $\begin{array}{c}1.5 \\
152\end{array}$ & & & & & & & & \\
\hline & $f$ & $\begin{array}{c}{\left[\begin{array}{ll}1.0 & 2.0\end{array}\right]} \\
{\left[\begin{array}{ll}1.7 & 2.7\end{array}\right]}\end{array}$ & & & & & & & & \\
\hline
\end{tabular}

1463 
1464 Table S4. Free parameters for the fits to the neutral and central cueing conditions of the four

1465 endogenous attention experiments. Bold values indicate the median and values within square

1466 brackets depict the $95 \% \mathrm{Cl}$ of the bootstrapped distribution of fitted parameters. $\min .=$ minimum;

1467 bw = bandwidth; amp. = amplitude .

1468

\begin{tabular}{|c|c|c|c|c|c|c|c|c|c|c|}
\hline \multirow{3}{*}{\multicolumn{2}{|c|}{$\begin{array}{l}\text { parameter } \\
\text { description }\end{array}$}} & \multicolumn{2}{|c|}{ Contrast gain } & \multicolumn{3}{|c|}{ Stimulus drive } & \multicolumn{4}{|c|}{ Broad gain profile } \\
\hline & & $g_{\sigma}$ & $m_{\sigma}$ & $\mathrm{t}_{\mathrm{T}}$ & $\mathrm{m}_{\mathrm{T}}$ & $\mathrm{b}_{\mathrm{T}}$ & $a_{\mathrm{B}}$ & $m_{B}$ & $b_{B}$ & $\gamma_{B}$ \\
\hline & & $\min$. & slope & SF peak & SF slope & SF bw & SF peak & SF slope & SF bw & $a m p$. \\
\hline \multirow{4}{*}{ 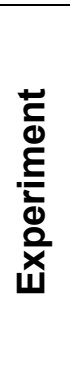 } & $g$ & $\begin{array}{c}\mathbf{1 . 6} \\
{\left[\begin{array}{ll}1.5 & 2.1\end{array}\right]}\end{array}$ & \multirow{4}{*}{$\begin{array}{c}\mathbf{- 0 . 0 9} \\
{[-0.1-0.06]}\end{array}$} & \multirow{4}{*}{$\begin{array}{c}3.4 \\
{[2.84 .0]}\end{array}$} & \multirow{4}{*}{$\begin{array}{c}-\mathbf{0 . 8} \\
{[-1.0-0.5]}\end{array}$} & \multirow{4}{*}{$\begin{array}{c}1.3 \\
{\left[\begin{array}{ll}1.1 & 1.6\end{array}\right]}\end{array}$} & \multirow{4}{*}{$\begin{array}{c}1.7 \\
{[0.52 .3]}\end{array}$} & \multirow{4}{*}{$\begin{array}{c}-\mathbf{0 . 2} \\
{[-0.3-0.1]}\end{array}$} & \multirow{4}{*}{$\begin{array}{c}\mathbf{4 . 5} \\
{[2.16 .6]}\end{array}$} & \multirow{4}{*}{$\begin{array}{c}3.1 \\
{[2.26 .2]}\end{array}$} \\
\hline & $\mathrm{h}$ & $\begin{array}{c}1.7 \\
{[1.51 .9]} \\
\end{array}$ & & & & & & & & \\
\hline & i & $\begin{array}{c}\mathbf{2 . 5} \\
{[2.22 .8]}\end{array}$ & & & & & & & & \\
\hline & j & $\begin{array}{c}\mathbf{1 . 7} \\
{\left[\begin{array}{ll}1.5 & 1.9\end{array}\right]}\end{array}$ & & & & & & & & \\
\hline
\end{tabular}


1470 Table S5. Stimulus parameters for each texture segmentation experiment.

1471

\begin{tabular}{|c|l|l|l|l|l|}
\hline Experiment & $\begin{array}{l}\text { Line spacing } \\
\text { horz } \times \text { vert }\end{array}$ & $\begin{array}{l}\text { Line size } \\
\text { width } \times \text { height }\end{array}$ & $\begin{array}{l}\text { Target size } \\
\text { width } \times \text { height }\end{array}$ & $\begin{array}{l}\text { Target } \\
\text { meridian }\end{array}$ & $\begin{array}{l}\text { Backward } \\
\text { mask }\end{array}$ \\
\hline $\mathrm{a}$ & $0.68^{\circ} \times 0.71^{\circ}$ & $0.1^{\circ} \times 0.4^{\circ}$ & $1.97^{\circ} \times 2.03^{\circ}$ & horizontal & yes \\
\hline $\mathrm{b}$ & $1.36^{\circ} \times 1.42^{\circ}$ & $0.2^{\circ} \times 0.8^{\circ}$ & $3.78^{\circ} \times 3.84^{\circ}$ & horizontal & yes \\
\hline $\mathrm{c}$ & $0.71^{\circ} \times 0.68^{\circ}$ & $0.1^{\circ} \times 0.4^{\circ}$ & $2.03^{\circ} \times 1.97^{\circ}$ & vertical & yes \\
\hline $\mathrm{d}$ & $1.43^{\circ} \times 1.37^{\circ}$ & $0.2^{\circ} \times 1^{\circ}$ & $4.09^{\circ} \times 3.97^{\circ}$ & horizontal & yes \\
\hline $\mathrm{e}$ & $0.68^{\circ} \times 0.71^{\circ}$ & $0.1^{\circ} \times 0.7^{\circ}$ & $2.28^{\circ} \times 2.34^{\circ}$ & horizontal & yes \\
\hline $\mathrm{f}$ & $0.68^{\circ} \times 0.71^{\circ}$ & $0.1^{\circ} \times 0.7^{\circ}$ & $2.28^{\circ} \times 2.34^{\circ}$ & horizontal & yes \\
\hline $\mathrm{g}$ & $0.68^{\circ} \times 0.71^{\circ}$ & $0.1^{\circ} \times 0.7^{\circ}$ & $2.28^{\circ} \times 2.34^{\circ}$ & horizontal & yes \\
\hline $\mathrm{h}$ & $0.46^{\circ} \times 0.46^{\circ}$ & $0.1^{\circ} \times 0.2^{\circ}$ & $1.34^{\circ} \times 1.34^{\circ}$ & intercardinal & no \\
\hline $\mathrm{i}$ & $0.91^{\circ} \times 0.91^{\circ}$ & $0.2^{\circ} \times 0.4^{\circ}$ & $2.47^{\circ} \times 2.47^{\circ}$ & intercardinal & no \\
\hline $\mathrm{j}$ & $0.4^{\circ} \times 0.4^{\circ}$ & $0.1^{\circ} \times 0.24^{\circ}$ & $1.03^{\circ} \times 1.84^{\circ}$ & intercardinal & no \\
\hline
\end{tabular}


1473 Table S6. Best-fitting parameters for acuity and contrast sensitivity tasks. min. = minimum; bw =

1474 bandwidth; amp. = amplitude; acuity = acuity experiment (6); CS = contrast sensitivity experiment

1475 (26). The 95\% confidence intervals show parameter values for fits to texture segmentation

1476 experiments, split by attention type.

1477

\begin{tabular}{|c|c|c|c|c|c|c|c|c|c|c|c|}
\hline \multirow{3}{*}{\multicolumn{2}{|c|}{$\begin{array}{l}\text { parameter } \\
\text { description }\end{array}$}} & \multicolumn{2}{|c|}{ Contrast gain } & \multicolumn{3}{|c|}{ Stimulus drive } & \multicolumn{5}{|c|}{ Attentional gain } \\
\hline & & $g_{\sigma}$ & $\mathrm{m}_{\sigma}$ & $t_{T}$ & $\mathrm{~m}_{\mathrm{T}}$ & $b_{T}$ & a & $\mathrm{m}$ & $b$ & $\gamma$ & $b_{\text {pos }}$ \\
\hline & & $\min$. & slope & SF peak & SF slope & SF bw & SF peak & SF slope & $S F b w$ & amp. & spread \\
\hline \multirow{3}{*}{ 이 } & $\begin{array}{r}95 \% \\
\mathrm{Cl} \\
\end{array}$ & [ 1.52 .8$]$ & {$\left[\begin{array}{lll}-0.07 & -0.05\end{array}\right]$} & {$\left[\begin{array}{ll}1.8 & 2.8\end{array}\right]$} & {$[-0.9-0.5]$} & [1.4 2.1] & [1.8 3.0] & {$\left[\begin{array}{lll}-0.3 & -0.01]\end{array}\right]$} & [1.5 4.8] & [3.0 7.8] & - \\
\hline & Acuity & 2 & -0.07 & 1.8 & -0.8 & 1.4 & 2.8 & -0.03 & 1.5 & 8 & 0.6 \\
\hline & CS & 2.5 & -0.03 & 1.1 & -0.2 & 1.4 & 1.5 & -0.01 & 2.3 & 1.5 & 5.2 \\
\hline \multirow{3}{*}{ 을 } & $\begin{array}{r}95 \% \\
\mathrm{Cl} \\
\end{array}$ & [ 1.5 2.8] & {$[-0.1-0.06]$} & {$\left[\begin{array}{lll}2.8 & 4.0\end{array}\right]$} & {$\left[\begin{array}{ll}-1.0 & -0.5\end{array}\right]$} & [1.1 1.6] & [0.5 2.3] & {$[-0.3-0.1]$} & [2.16.6] & [2.2 6.2] & - \\
\hline & Acuity & 1.5 & -0.09 & 3 & -0.9 & 1.1 & 2 & -0.3 & 5.3 & 15.0 & 0.6 \\
\hline & CS & 2.5 & -0.04 & 1.1 & -0.2 & 1.5 & 2.6 & -0.2 & 4.2 & 1.2 & 5.2 \\
\hline
\end{tabular}

1478 